\title{
The diversity of Pseudomonas species isolated from fish farms in Turkey
}

\author{
Muhammed Duman ${ }^{\mathrm{a}}$, Magdalena Mulet ${ }^{\mathrm{b}}$, Soner Altun ${ }^{\mathrm{a}}$, Izzet Burcin Saticioglu ${ }^{\mathrm{c}}$, \\ Burak Ozdemir ${ }^{\mathrm{a}}$, Nihed Ajmi ${ }^{\mathrm{a}}$, Jorge Lalucat ${ }^{\mathrm{b}, \mathrm{d}}$, Elena García-Valdés ${ }^{\mathrm{b}, \mathrm{d}, \text {, }}$ \\ ${ }^{a}$ Department of Aquatic Animal Diseases, Faculty of Veterinary Medicine, Bursa Uludag University, 16059 Bursa, Turkey \\ ${ }^{\mathrm{b}}$ Microbiologia, Departament de Biologia, Edifici Guillem Colom, Universitat de les Illes Balears, Campus UIB, 07122 Palma de Mallorca, Spain \\ ${ }^{c}$ Department of Aquatic Animal Diseases, Faculty of Veterinary Medicine, Erciyes University, 38280 Kayseri, Turkey \\ d Institut Mediterrani d'Estudis Avançats (IMEDEA, CSIC-UIB), Campus UIB, 07122 Palma de Mallorca, Spain
}

\section{A R T I C L E I N F O}

\section{Keywords:}

Pseudomonas

Aquaculture

Water

Rainbow trout

Onchorhynchus mykiss

\begin{abstract}
A B S T R A C T
The prevalence of Pseudomonas species in freshwater fish and water from aquaculture farms in Turkey was determined on a monthly basis, in the period covering 2013-2017 and two seasons of 2018. The farms included in the study were located in six different regions of Turkey. A total of 90 Pseudomonas strains were isolated from water and diseased and healthy fish, and were classified under 20 species. The phenotypic characterization of the strains was based on oxidase and catalase activities, haemolysis, tolerance to temperature and sodium chloride, the production of fluorescent pigments and antibiotic resistance spectra. The phylogenetic identification of the 90 isolates was performed by a 4-gene multilocus sequence analysis, based on the partial sequencing of the $16 \mathrm{~S}$ rRNA, $g y r B$, rpoB and $r p o D$ genes. This is the first report on the isolation of several Pseudomonas species, namely, P. brenneri, P. defensor, P. haemolytica, P. lactis, P. lundensis, P. lurida, P. mandelii, P. meridiana, P. migulae, $P$. proteolytica, $P$. simiae, and $P$. weihenstephanensis, from freshwater salmonid fish. Two of these species, $P$. haemolytica and $P$. lactis have been isolated for the first time from fish farms, an environment quite different from their original isolation source, raw milk. Furthermore, seven putative new Pseudomonas species were isolated from water and farmed rainbow trout. During the 2013 to 2018 period, several Pseudomonas species were detected to have spread from the Aegean and Central Anatolia regions to the Eastern Anatolia and Black Sea regions.
\end{abstract}

\section{Introduction}

A large number of Pseudomonas species are pathogenic for humans, animals and aquatic organisms (Nixon et al., 2001)). Pseudomonas species have been described as one of the most common bacterial infectious agents of cultured fish and have been reported to cause stress-related diseases in freshwater fish, especially under farming conditions (López et al., 2012; Derome et al., 2016). Several studies have reported almost $100 \%$ mortality due to infection with Pseudomonas spp. in rainbow trout, sea bream, sea bass and ayu in farm settings (López et al., 2012; Pridgeon, 2012; Thomas et al., 2014; Derome et al., 2016). Although Pseudomonas spp. have been described as opportunistic pathogens, many species have also been identified as the primary pathogen of several diseases in farmed fish, including $P$. aeruginosa, $P$. anguilliseptica, $P$. baetica, P. chlororaphis, P. fluorescens, P. koreensis, P. luteola, P. plecoglossicida, P. pseudoalcaligenes and P. putida (Altinok et al., 2006; Altinok et al., 2007; López et al., 2012; Thomas et al., 2014; Austin and Austin, 2016; Derome et al., 2016). Recently, a new species, named P. tructae, was isolated from rainbow trout kidney (Oh et al., 2019). The pathogenicity of many other species, including among others $P$. asiatica, $P$. brenneri, $P$. lactis, $P$. lundensis, $P$. lurida, $P$. mandelii, $P$. meridiana, $P$. migulae, $P$. proteolytica, $P$. simiae and $P$. weihenstephanensis, for farmed fish has not yet been established (Austin and Austin, 2016). In fact, noninformation on the exact cause of disease hinders taking preventative measures and using disease control agents. To date, only two formalin-inactivated vaccines have been developed against P. anguillaseptica and P. plecoglossicida (Austin and Austin, 2016). On the other hand, Pseudomonas species have common use as probiotic organisms in aquaculture, because they can suppress major mycological and bacterial agents, such as Saprolegnia and aeromonads, and probably other emerging diseases (Das et al., 2006; Liu et al., 2015).

The identification of Pseudomonas species, and in particular their

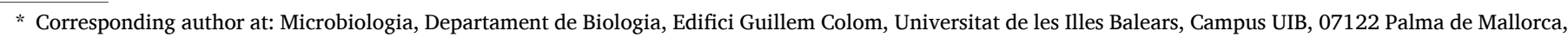
Spain.

E-mail address: elena.garciavaldes@uib.es (E. García-Valdés).
} 
differentiation from other closest genera, require the performance of multiple laboratory tests and a detailed molecular analysis (Mulet et al., 2012; Palleroni, 2015). The main phenotypic characteristics of the genus are pigment production, such as fluorescein or pyocyanin, and oxidasepositivity, but the identification of non-pigmented and/or oxidasenegative species by biochemical methods alone is difficult (Palleroni, 2015). Molecular methods used for the identification of Pseudomonas species include 16S rRNA sequencing for genus identification and multilocus sequence analysis (MLSA) for species affiliation (Baumann et al., 1983; Ait Tayeb et al., 2005; Mulet et al., 2009, 2012; Gomila et al., 2015).

In the present study, with an aim to determine the prevalence of Pseudomonas species, salmonid freshwater fish farmed in six different regions of Turkey were surveyed on a monthly basis in a period covering 2013-2017 and two seasons of 2018. Bacteriological examinations were performed on both seemingly healthy fish and symptomatic or moribund fish. In total, 90 Pseudomonas strains were isolated and taxonomically characterized by extensive phenotypic analyses and a multilocus sequencing approach, based on the analyses of partial sequences of the $16 \mathrm{~S}$ rRNA, gyrB, $r p o B$ and $r p o D$ genes. To our knowledge, this the first time that $P$. brenneri, $P$. haemolytica, $P$. lactis, $P$. lundensis, $P$. lurida, $P$. mandelii, $P$. meridiana, $P$. migulae, $P$. proteolytica, $P$. simiae, and $P$. weihenstephanensis have been recovered from freshwater salmonid fish. In addition, strains of at least seven putative novel species were isolated and taxonomically characterized using a polyphasic approach. This study provides an update of the known diversity, abundance and specific habitat (farm) distribution of Pseudomonas species.

\section{Material and methods}

\subsection{Bacterial isolation}

Aquaculture farms with high production capacities, using spring water, stream water and dammed lake water, and located in six different regions of Turkey (Marmara, Aegean, Central Anatolia, Black Sea, Mediterranean, and Eastern Anatolia), were included in this study. Sampling was done mainly from rainbow trouts (Oncorhynchus mykiss, Walbaum, 1792) and from other trout species, including the Black sea trout, Brook trout, and Brown trout (Salmo trutta labrax, Pallas, 1814; Salvelinus fontinalis, Mitchill, 1814; and Salmo trutta magrostigma, Dumeril, 1858; respectively). Samples were taken from fish, eggs or milt in the spawning period, and also from alevins. As part of a health surveillance study, 100 rainbow trouts, which were either subclinically infected, clinically infected or moribund, were randomly collected on a monthly basis, during a period covering 2013-2017 and two seasons in 2018. Fish farms located in the Black Sea region produce rainbow trout and local trout species. The local trout species (Salmo trutta group) farmed in the Black Sea region were sampled twice a year, during the period from 2013 to 2017, such that a total of 1000 Salmo trutta were sampled throughout the surveillance period.

More than four thousand fish were analysed during the five-year surveillance period (2013-2017). Bacteria were isolated from both seemingly healthy fish (based on farmer observations, and external and internal examinations) and from diseased fish, either symptomatic or moribund. In farms with only seemingly healthy fish, sampling involved fish of all weights, from egg to brood stock, regardless of their health situation and the farmers' suggestions.

Furthermore, eggs or milt were also sampled in the spawning period, which covered the period from November to March in breeding stocks farmed under a natural light-dark regime and also the summer season in stocks exposed to photoperiod manipulations.

Alevin or yolk-sac larvae were sampled twice a year, such that a hundred were sampled each time. From 2013 to 2017, a total of 200 fish (alevin or yolk-sac larvae) were sampled each year. The samples were collected aseptically from the yolk-sac of alevin or larvae with a sterile loop. When the yolk-sac was not apparent in small fish weighing around
$0.5 \mathrm{~g}$, samples were taken from the surface and abdominal cavity.

Fish were classified according to health status, region, cohort, weight, and month. Samples from diseased fish were taken aseptically with sterile loops and swabs from skin lesions, and the liver, kidney, spleen, yolk sac, ovary and eggs. Samples were plated on tryptic soy agar (Merck, 105,458, TSA), and blood agar (BA; with 5\% sheep blood). In the event of the occurrence of mass fish death on a farm, in addition to the fish samples, water samples were also collected from pond inlets, outlets, and benthic zones to monitor the dissemination of the agents. Water samples were taken from fish farms three times (from ten different ponds) in 2014, twice (from six different ponds and also one benthic zone) in 2015, and three times (from six different ponds) in 2016 and 2017. In total, 60 pond water samples were analysed for the detection of fish pathogens from 2014 to 2017. In 2018, the sampling in Sivas was repeated in April, due to a disease case, and another health control sampling was performed in December in Mugla.

According to the health surveillance program, fish samples were also used for investigating the presence of Aeromonas and Flavobacterium species. Isolation and identification were performed by following the protocols described by Loch et al. (2013) for Flavobacterium spp. Special attention was paid to the hatcheries, where these genera had been detected previously (Duman et al., 2017, 2018; Saticioglu et al., 2018).

Samplings were carried out according to the guidelines for the diagnosis of fish diseases and in compliance with the international guidelines for animal welfare and guidelines for aquatic animal health surveillance (OIE-Office International Des E, 2000; Austin and NewajFyzul, 2017). Water samples were collected into sterile bottles and transported to the laboratory on ice. Samples were serially diluted and concentrated on nitrocellulose membrane filters $(0.45-\mu \mathrm{m}$ pore size; Millipore) by passing $100 \mathrm{ml}$ of each dilution through the filter using the membrane filtration technique. The filters were then placed onto Pseudomonas F agar plates (Merck, 110,989). The spread plate technique was also employed by spreading $100 \mu \mathrm{l}$ of pond water samples onto tryptic soy agar (TSA), blood agar (BA) and Pseudomonas F agar, and bacterial colonies were selected by fluorescent pigment production, morphology, and phenotypical tests (Khan et al., 2010; Palleroni, 2015).

All isolates were sub-cultured on Pseudomonas F medium for two days at $28^{\circ} \mathrm{C}$ to ensure purity. Furthermore, all isolates were cultured on tryptic soy broth (TSB) (Merck, 105,459) at $28{ }^{\circ} \mathrm{C}$ for $24-48 \mathrm{~h}$, and pure cultures were supplemented with $20 \%$ glycerol and kept at $-80{ }^{\circ} \mathrm{C}$ for long-term storage. The strains used in this study, and their origins are listed in Table 1.

\subsection{Physiological and biochemical characteristics}

The biochemical characteristics of the isolates were determined using conventional microbial tests, including the assessment of colony morphology, Gram staining, oxidase and catalase activities, growth on different media, and to different temperature conditions (Palleroni, 2015; Austin and Austin, 2016). Bacterial motility was observed by the hanging drop technique and checked on sulfide-indole-motility (SIM) medium (Merck, 1,054,700,500) (Austin and Austin, 2016). The pellicle formation ability of the Pseudomonas isolates was detected as described by Palleroni (2015), and haemolytic characteristics were assessed by plating on blood agar containing 5\% sheep blood. Growth temperatures $\left(4,25,37,42\right.$, and $\left.45^{\circ} \mathrm{C}\right)$ were determined in TSB medium, and growth in the presence of $\mathrm{NaCl}(0-10 \% w / v)$ was tested in TSB medium containing different concentrations of $\mathrm{NaCl}$. Proteinase production (hydrolysis) was tested by plating on nutrient agar containing $2.5 \%$ skimmed milk for $72-96 \mathrm{~h}$ at $28{ }^{\circ} \mathrm{C}$. Proteolytic activity was estimated based on the size of the clear zone around the colony (Vazquez et al., 1995). The bacteria were cultured routinely on Pseudomonas F agar medium at $28^{\circ} \mathrm{C}$. The production of fluorescent pigments was tested on King B medium (Lamichhane and Varvaro, 2013). Plates were viewed on an ultraviolet (UV) transilluminator, and luminescence was determined for each culture at a wavelength of $360 \mathrm{~nm}$. Luminescence strength was 
Table 1

Strains used in this study and their origins.

\begin{tabular}{|c|c|c|c|c|c|c|c|c|c|}
\hline Strains & Fish species & $\begin{array}{l}\text { Fish } \\
\text { weight (g) }\end{array}$ & $\begin{array}{l}\text { Isolation } \\
\text { year }\end{array}$ & $\begin{array}{l}\text { Isolation } \\
\text { month }\end{array}$ & Town & City & Region & Fish health status* & Accompanying species \\
\hline P1 & $\begin{array}{l}\text { Oncorhynchus } \\
\text { mykiss }\end{array}$ & 10 & 2013 & December & Uzunyayla & Kayseri & $\begin{array}{l}\text { Central } \\
\text { Anatolia }\end{array}$ & & \\
\hline P2 & $\begin{array}{l}\text { Oncorhynchus } \\
\text { mykiss }\end{array}$ & 10 & 2013 & December & Uzunyayla & Kayseri & $\begin{array}{l}\text { Central } \\
\text { Anatolia }\end{array}$ & & \\
\hline P3a & $\begin{array}{l}\text { Oncorhynchus } \\
\text { mykiss }\end{array}$ & 10 & 2013 & December & Uzunyayla & Kayseri & $\begin{array}{l}\text { Central } \\
\text { Anatolia }\end{array}$ & & \\
\hline P3b & $\begin{array}{l}\text { Oncorhynchus } \\
\text { mykiss }\end{array}$ & 10 & 2013 & December & Uzunyayla & Kayseri & $\begin{array}{l}\text { Central } \\
\text { Anatolia }\end{array}$ & & \\
\hline P5 & $\begin{array}{l}\text { Oncorhynchus } \\
\text { mykiss }\end{array}$ & 0.4 & 2013 & December & Uzunyayla & Kayseri & $\begin{array}{l}\text { Central } \\
\text { Anatolia }\end{array}$ & & \\
\hline P7 & $\begin{array}{l}\text { Oncorhynchus } \\
\text { mykiss }\end{array}$ & 0.4 & 2013 & December & Uzunyayla & Kayseri & $\begin{array}{l}\text { Central } \\
\text { Anatolia }\end{array}$ & & \\
\hline P9 & $\begin{array}{l}\text { Oncorhynchus } \\
\text { mykiss }\end{array}$ & 0.5 & 2013 & December & Uzunyayla & Kayseri & $\begin{array}{l}\text { Central } \\
\text { Anatolia }\end{array}$ & & \\
\hline P10 & $\begin{array}{l}\text { Oncorhynchus } \\
\text { mykiss }\end{array}$ & 0.5 & 2013 & December & Uzunyayla & Kayseri & $\begin{array}{l}\text { Central } \\
\text { Anatolia }\end{array}$ & & \\
\hline P11 & water & water & 2014 & December & Uzunyayla & Kayseri & $\begin{array}{l}\text { Central } \\
\text { Anatolia }\end{array}$ & & \\
\hline P16 & $\begin{array}{l}\text { Oncorhynchus } \\
\text { mykiss }\end{array}$ & $30-40$ & 2014 & December & Uzunyayla & Kayseri & $\begin{array}{l}\text { Central } \\
\text { Anatolia }\end{array}$ & & \\
\hline P21 & $\begin{array}{l}\text { Oncorhynchus } \\
\text { mykiss }\end{array}$ & 10 & 2013 & November & Ören & Mugla & Aegean & & \\
\hline P24-1 & $\begin{array}{l}\text { Oncorhynchus } \\
\text { mykiss }\end{array}$ & 0.5 & 2013 & December & Uzunyayla & Kayseri & $\begin{array}{l}\text { Central } \\
\text { Anatolia }\end{array}$ & & \\
\hline P24-2 & $\begin{array}{l}\text { Oncorhynchus } \\
\text { mykiss }\end{array}$ & 0.5 & 2013 & December & Uzunyayla & Kayseri & $\begin{array}{l}\text { Central } \\
\text { Anatolia }\end{array}$ & & \\
\hline P25 & $\begin{array}{l}\text { Oncorhynchus } \\
\text { mykiss }\end{array}$ & 0.5 & 2013 & December & Uzunyayla & Kayseri & $\begin{array}{l}\text { Central } \\
\text { Anatolia }\end{array}$ & & \\
\hline P27 & $\begin{array}{l}\text { Oncorhynchus } \\
\text { mykiss }\end{array}$ & 10 & 2013 & December & Uzunyayla & Kayseri & $\begin{array}{l}\text { Central } \\
\text { Anatolia }\end{array}$ & & \\
\hline P29 & $\begin{array}{l}\text { Oncorhynchus } \\
\text { mykiss }\end{array}$ & 0.4 & 2013 & December & Uzunyayla & Kayseri & $\begin{array}{l}\text { Central } \\
\text { Anatolia }\end{array}$ & & \\
\hline P30 & $\begin{array}{l}\text { Oncorhynchus } \\
\text { mykiss }\end{array}$ & 10 & 2013 & December & Uzunyayla & Kayseri & $\begin{array}{l}\text { Central } \\
\text { Anatolia }\end{array}$ & & \\
\hline P31 & $\begin{array}{l}\text { Oncorhynchus } \\
\text { mykiss }\end{array}$ & 10 & 2013 & December & Uzunyayla & Kayseri & $\begin{array}{l}\text { Central } \\
\text { Anatolia }\end{array}$ & & \\
\hline P34 & $\begin{array}{l}\text { Oncorhynchus } \\
\text { mykiss }\end{array}$ & 10 & 2013 & December & Uzunyayla & Kayseri & $\begin{array}{l}\text { Central } \\
\text { Anatolia }\end{array}$ & Dorsal lesion & \\
\hline P42 & $\begin{array}{l}\text { Oncorhynchus } \\
\text { mykiss }\end{array}$ & $0.1-0.5$ & 2014 & March & Uzunyayla & Kayseri & $\begin{array}{l}\text { Central } \\
\text { Anatolia }\end{array}$ & & $\begin{array}{l}\text { Flavobacterium } \\
\text { psychrophilum }^{b} / \\
\text { branchiarum }^{a}\end{array}$ \\
\hline P43 & $\begin{array}{l}\text { Oncorhynchus } \\
\text { mykiss }\end{array}$ & $0.5-1$ & 2014 & March & Uzunyayla & Kayseri & $\begin{array}{l}\text { Central } \\
\text { Anatolia }\end{array}$ & & $\begin{array}{l}\text { Flavobacterium } \\
\text { psychrophilum }^{a} / \\
\text { branchiarum }^{a}\end{array}$ \\
\hline P44 & $\begin{array}{l}\text { Oncorhynchus } \\
\text { mykiss }\end{array}$ & $3-5$ & 2013 & July & Uzunyayla & Kayseri & $\begin{array}{l}\text { Central } \\
\text { Anatolia }\end{array}$ & & \\
\hline P45 & $\begin{array}{l}\text { Oncorhynchus } \\
\text { mykiss }\end{array}$ & 8 & 2013 & July & Uzunyayla & Kayseri & $\begin{array}{l}\text { Central } \\
\text { Anatolia }\end{array}$ & & \\
\hline P46 & $\begin{array}{l}\text { Oncorhynchus } \\
\text { mykiss }\end{array}$ & 8 & 2013 & July & Uzunyayla & Kayseri & $\begin{array}{l}\text { Central } \\
\text { Anatolia }\end{array}$ & & \\
\hline P47 & $\begin{array}{l}\text { Oncorhynchus } \\
\text { mykiss }\end{array}$ & 8 & 2013 & July & Uzunyayla & Kayseri & $\begin{array}{l}\text { Central } \\
\text { Anatolia }\end{array}$ & & \\
\hline P48 & $\begin{array}{l}\text { Oncorhynchus } \\
\text { mykiss }\end{array}$ & 20 & 2013 & July & Su Şehri & Sivas & $\begin{array}{l}\text { Central } \\
\text { Anatolia }\end{array}$ & Dorsal lesion & \\
\hline P49 & $\begin{array}{l}\text { Oncorhynchus } \\
\text { mykiss }\end{array}$ & $0.2-0.5$ & 2013 & June & Uzunyayla & Kayseri & $\begin{array}{l}\text { Central } \\
\text { Anatolia }\end{array}$ & Dorsal lesion & \\
\hline P50 & $\begin{array}{l}\text { Oncorhynchus } \\
\text { mykiss }\end{array}$ & $0.2-0.5$ & 2013 & June & Uzunyayla & Kayseri & $\begin{array}{l}\text { Central } \\
\text { Anatolia }\end{array}$ & Dorsal lesion & \\
\hline P51 & $\begin{array}{l}\text { Oncorhynchus } \\
\text { mykiss }\end{array}$ & 3000 & 2013 & July & Su Şehri & Sivas & $\begin{array}{l}\text { Central } \\
\text { Anatolia }\end{array}$ & Dorsal lesion & \\
\hline P52 & $\begin{array}{l}\text { Oncorhynchus } \\
\text { mykiss }\end{array}$ & 3000 & 2013 & July & Uzunyayla & Kayseri & $\begin{array}{l}\text { Central } \\
\text { Anatolia }\end{array}$ & Moribund fish & \\
\hline P54 & $\begin{array}{l}\text { Oncorhynchus } \\
\text { mykiss }\end{array}$ & 3000 & 2013 & July & Uzunyayla & Kayseri & $\begin{array}{l}\text { Central } \\
\text { Anatolia }\end{array}$ & Moribund fish & \\
\hline P55 & $\begin{array}{l}\text { Oncorhynchus } \\
\text { mykiss }\end{array}$ & 3000 & 2013 & July & Uzunyayla & Kayseri & $\begin{array}{l}\text { Central } \\
\text { Anatolia }\end{array}$ & Moribund fish & \\
\hline P56 & $\begin{array}{l}\text { Oncorhynchus } \\
\text { mykiss }\end{array}$ & 3000 & 2013 & July & Uzunyayla & Kayseri & $\begin{array}{l}\text { Central } \\
\text { Anatolia }\end{array}$ & Moribund fish & \\
\hline P57 & $\begin{array}{l}\text { Oncorhynchus } \\
\text { mykiss }\end{array}$ & 3000 & 2013 & July & Uzunyayla & Kayseri & $\begin{array}{l}\text { Central } \\
\text { Anatolia }\end{array}$ & Moribund fish & \\
\hline P58 & $\begin{array}{l}\text { Oncorhynchus } \\
\text { mykiss }\end{array}$ & 3000 & 2013 & July & Uzunyayla & Kayseri & $\begin{array}{l}\text { Central } \\
\text { Anatolia }\end{array}$ & Moribund fish & \\
\hline P59 & & 3000 & 2013 & July & Uzunyayla & Kayseri & & Moribund fish & \\
\hline
\end{tabular}


Table 1 (continued)

\begin{tabular}{|c|c|c|c|c|c|c|c|c|c|}
\hline Strains & Fish species & $\begin{array}{l}\text { Fish } \\
\text { weight }(g)\end{array}$ & $\begin{array}{l}\text { Isolation } \\
\text { year }\end{array}$ & $\begin{array}{l}\text { Isolation } \\
\text { month }\end{array}$ & Town & City & Region & Fish health status* & Accompanying species \\
\hline & $\begin{array}{l}\text { Oncorhynchus } \\
\text { mykiss }\end{array}$ & & & & & & $\begin{array}{l}\text { Central } \\
\text { Anatolia }\end{array}$ & & \\
\hline P66 & $\begin{array}{l}\text { Oncorhynchus } \\
\text { mykiss }\end{array}$ & 0.3 & 2014 & February & Ören & Mugla & Aegean & & \\
\hline P67 & $\begin{array}{l}\text { Oncorhynchus } \\
\text { mykiss }\end{array}$ & 0.3 & 2014 & February & Ören & Mugla & Aegean & & \\
\hline P68b & $\begin{array}{l}\text { Oncorhynchus } \\
\text { mykiss }\end{array}$ & 0.3 & 2014 & February & Ören & Mugla & Aegean & & \\
\hline P72 & water & water & 2014 & January & Su Şehri & sivas & $\begin{array}{l}\text { Central } \\
\text { Anatolia }\end{array}$ & & \\
\hline P73 & water & water & 2014 & January & Uzunyayla & Kayseri & $\begin{array}{l}\text { Central } \\
\text { Anatolia }\end{array}$ & & \\
\hline P91 & $\begin{array}{l}\text { Oncorhynchus } \\
\text { mykiss }\end{array}$ & $3-5$ & 2013 & July & Uzunyayla & Kayseri & $\begin{array}{l}\text { Central } \\
\text { Anatolia }\end{array}$ & & \\
\hline P93 & $\begin{array}{l}\text { Oncorhynchus } \\
\text { mykiss }\end{array}$ & 8 & 2013 & July & Uzunyayla & Kayseri & $\begin{array}{l}\text { Central } \\
\text { Anatolia }\end{array}$ & & \\
\hline P94 & $\begin{array}{l}\text { Oncorhynchus } \\
\text { mykiss }\end{array}$ & 8 & 2013 & July & Uzunyayla & Kayseri & $\begin{array}{l}\text { Central } \\
\text { Anatolia }\end{array}$ & & \\
\hline P95 & $\begin{array}{l}\text { Oncorhynchus } \\
\text { mykiss }\end{array}$ & 8 & 2013 & July & Uzunyayla & Kayseri & $\begin{array}{l}\text { Central } \\
\text { Anatolia }\end{array}$ & & \\
\hline P97 & $\begin{array}{l}\text { Oncorhynchus } \\
\text { mykiss }\end{array}$ & 3000 & 2013 & July & Uzunyayla & Kayseri & $\begin{array}{l}\text { Central } \\
\text { Anatolia }\end{array}$ & Moribund fish & \\
\hline P99 & $\begin{array}{l}\text { Oncorhynchus } \\
\text { mykiss }\end{array}$ & 3000 & 2013 & July & Uzunyayla & Kayseri & $\begin{array}{l}\text { Central } \\
\text { Anatolia }\end{array}$ & Moribund fish & \\
\hline P100 & $\begin{array}{l}\text { Oncorhynchus } \\
\text { mykiss }\end{array}$ & 3000 & 2013 & July & Uzunyayla & Kayseri & $\begin{array}{l}\text { Central } \\
\text { Anatolia }\end{array}$ & Moribund fish & \\
\hline P101 & $\begin{array}{l}\text { Oncorhynchus } \\
\text { mykiss }\end{array}$ & 3000 & 2013 & July & Uzunyayla & Kayseri & $\begin{array}{l}\text { Central } \\
\text { Anatolia }\end{array}$ & Moribund fish & \\
\hline P102 & $\begin{array}{l}\text { Oncorhynchus } \\
\text { mykiss }\end{array}$ & 20 & 2013 & July & Su Şehri & Sivas & $\begin{array}{l}\text { Central } \\
\text { Anatolia }\end{array}$ & Dorsal lesion & \\
\hline P106 & $\begin{array}{l}\text { Oncorhynchus } \\
\text { mykiss }\end{array}$ & 20 & 2013 & July & Su Şehri & Sivas & $\begin{array}{l}\text { Central } \\
\text { Anatolia }\end{array}$ & Dorsal lesion & \\
\hline P109 & $\begin{array}{l}\text { Oncorhynchus } \\
\text { mykiss }\end{array}$ & $60-70$ & 2013 & December & Ören & Mugla & Aegean & & \\
\hline P114 & $\begin{array}{l}\text { Oncorhynchus } \\
\text { mykiss }\end{array}$ & $0.5-1$ & 2018 & December & Ören & Mugla & Aegean & & \\
\hline P115 & $\begin{array}{l}\text { Oncorhynchus } \\
\text { mykiss }\end{array}$ & $0.5-1$ & 2018 & April & Su Şehri & Sivas & $\begin{array}{l}\text { Central } \\
\text { Anatolia }\end{array}$ & $\begin{array}{l}\text { Loss of appetite, anemia, } \\
\text { dorsal fin erosion }\end{array}$ & \\
\hline P116 & $\begin{array}{l}\text { Oncorhynchus } \\
\text { mykiss }\end{array}$ & $0.5-1$ & 2018 & April & Su Şehri & Sivas & $\begin{array}{l}\text { Central } \\
\text { Anatolia }\end{array}$ & $\begin{array}{l}\text { Loss of appetite, anemia, } \\
\text { dorsal fin erosion }\end{array}$ & \\
\hline P117 & $\begin{array}{l}\text { Oncorhynchus } \\
\text { mykiss }\end{array}$ & $0.5-1$ & 2018 & April & Su Şehri & Sivas & $\begin{array}{l}\text { Central } \\
\text { Anatolia }\end{array}$ & $\begin{array}{l}\text { Loss of appetite, anemia, } \\
\text { dorsal fin erosion }\end{array}$ & \\
\hline $\mathrm{P} 121$ & $\begin{array}{l}\text { Oncorhynchus } \\
\text { mykiss }\end{array}$ & 0.1 & 2017 & January & Ören & Mugla & Aegean & & Flavobacterium frigidimaris ${ }^{a}$ \\
\hline P122 & $\begin{array}{l}\text { Oncorhynchus } \\
\text { mykiss }\end{array}$ & 0.1 & 2017 & January & Ören & Mugla & Aegean & & Flavobacterium frigidimaris ${ }^{a}$ \\
\hline P123 & $\begin{array}{l}\text { Oncorhynchus } \\
\text { mykiss }\end{array}$ & 0.1 & 2017 & January & Ören & Mugla & Aegean & & Flavobacterium frigidimaris $^{a}$ \\
\hline P124 & $\begin{array}{l}\text { Oncorhynchus } \\
\text { mykiss }\end{array}$ & egg & 2017 & January & Ören & Mugla & Aegean & & $\begin{array}{l}\text { Flavobacterium } \\
\text { oncorhynchi }^{a} / \text { glaciei }^{a}\end{array}$ \\
\hline P131 & $\begin{array}{l}\text { Oncorhynchus } \\
\text { mykiss }\end{array}$ & $15-20$ & 2017 & January & Dalaman & Mugla & Aegean & & \\
\hline $\mathrm{P} 132$ & $\begin{array}{l}\text { Oncorhynchus } \\
\text { mykiss }\end{array}$ & $0.2-0.3$ & 2017 & January & Dalaman & Mugla & Aegean & & $\begin{array}{l}\text { Flavobacterium } \\
\text { psychrophilum }^{b} / \\
\text { branchiarum }^{a}\end{array}$ \\
\hline P135 & $\begin{array}{l}\text { Oncorhynchus } \\
\text { mykiss }\end{array}$ & 0.1 & 2017 & January & Dalaman & Mugla & Aegean & & $\begin{array}{l}\text { Flavobacterium tructae }^{a} / \\
\text { tiangeerense }^{a}\end{array}$ \\
\hline P137 & $\begin{array}{l}\text { Oncorhynchus } \\
\text { mykiss }\end{array}$ & 0.1 & 2017 & January & Ören & Mugla & Aegean & & Flavobacterium spp. $^{a}$ \\
\hline P138 & $\begin{array}{l}\text { Oncorhynchus } \\
\text { mykiss }\end{array}$ & 0.1 & 2017 & January & Ören & Mugla & Aegean & High mortality & Flavobacterium spp. $^{a}$ \\
\hline P139 & $\begin{array}{l}\text { Oncorhynchus } \\
\text { mykiss }\end{array}$ & 0.1 & 2017 & January & Ören & Mugla & Aegean & High mortality & Flavobacterium spp. $^{a}$ \\
\hline P141 & $\begin{array}{l}\text { Oncorhynchus } \\
\text { mykiss }\end{array}$ & 0.1 & 2017 & January & Ören & Mugla & Aegean & High mortality & Flavobacterium spp. $^{\text {a }}$ \\
\hline P147 & $\begin{array}{l}\text { Oncorhynchus } \\
\text { mykiss }\end{array}$ & 0.1 & 2017 & January & Uzunyayla & Kayseri & $\begin{array}{l}\text { Central } \\
\text { Anatolia }\end{array}$ & & Flavobacterium spp. $^{a}$ \\
\hline P152 & $\begin{array}{l}\text { Oncorhynchus } \\
\text { mykiss }\end{array}$ & yolk sac & 2017 & January & Uzunyayla & Kayseri & $\begin{array}{l}\text { Central } \\
\text { Anatolia }\end{array}$ & & \\
\hline P154a & $\begin{array}{l}\text { Oncorhynchus } \\
\text { mykiss }\end{array}$ & 0.1 & 2017 & January & Çağlayan & Rize & $\begin{array}{l}\text { Black Sea } \\
\text { region }\end{array}$ & & Flavobacterium collinsii $^{a}$ \\
\hline P154b & $\begin{array}{l}\text { Oncorhynchus } \\
\text { mykiss }\end{array}$ & 0.1 & 2017 & January & Çağlayan & Rize & $\begin{array}{l}\text { Black Sea } \\
\text { region }\end{array}$ & & Flavobacterium collinsii ${ }^{a}$ \\
\hline P155 & $\begin{array}{l}\text { Oncorhynchus } \\
\text { mykiss }\end{array}$ & 0.1 & 2017 & January & Çağlayan & Rize & $\begin{array}{l}\text { Black Sea } \\
\text { region }\end{array}$ & Abnormal swiming & Flavobacterium collinsii ${ }^{a}$ \\
\hline
\end{tabular}


Table 1 (continued)

\begin{tabular}{|c|c|c|c|c|c|c|c|c|c|}
\hline Strains & Fish species & $\begin{array}{l}\text { Fish } \\
\text { weight }(g)\end{array}$ & $\begin{array}{l}\text { Isolation } \\
\text { year }\end{array}$ & $\begin{array}{l}\text { Isolation } \\
\text { month }\end{array}$ & Town & City & Region & Fish health status* & Accompanying species \\
\hline P156 & $\begin{array}{l}\text { Salvelinus } \\
\text { fontinalis }\end{array}$ & egg & 2017 & January & Çağlayan & Rize & $\begin{array}{l}\text { Black Sea } \\
\text { region }\end{array}$ & & Flavobacterium collinsii ${ }^{a}$ \\
\hline P158 & $\begin{array}{l}\text { Oncorhynchus } \\
\text { mykiss }\end{array}$ & 0.1 & 2017 & January & Keban & Elazı ğ & $\begin{array}{l}\text { Eastern } \\
\text { region }\end{array}$ & & \\
\hline P162 & $\begin{array}{l}\text { Oncorhynchus } \\
\text { mykiss }\end{array}$ & yolk-sac & 2017 & January & Keban & Elazig & $\begin{array}{l}\text { Eastern } \\
\text { region }\end{array}$ & & \\
\hline P163 & $\begin{array}{l}\text { Salmo trutta } \\
\text { magrostigma }\end{array}$ & yolk-sac & 2017 & January & Çamlıhemşin & Rize & $\begin{array}{l}\text { Black Sea } \\
\text { region }\end{array}$ & & Flavobacterium collinsii ${ }^{a}$ \\
\hline P164 & $\begin{array}{l}\text { Salmo trutta } \\
\text { magrostigma }\end{array}$ & egg & 2017 & January & Çamlıhemşin & Rize & $\begin{array}{l}\text { Black Sea } \\
\text { region }\end{array}$ & & \\
\hline P166 & $\begin{array}{l}\text { Salvelinus. } \\
\text { fontinalis }\end{array}$ & 0.2 & 2017 & January & Çamlıhemşin & Rize & $\begin{array}{l}\text { Black Sea } \\
\text { region }\end{array}$ & & \\
\hline P167 & Salmo trutta labrax & 0.2 & 2017 & January & Çamlıhemşin & Rize & $\begin{array}{l}\text { Black Sea } \\
\text { region }\end{array}$ & & Flavobacterium piscis ${ }^{a}$ \\
\hline P168 & $\begin{array}{l}\text { Salvelinus } \\
\text { fontinalis }\end{array}$ & 1500 & 2017 & January & Çamlıhemşin & Rize & $\begin{array}{l}\text { Black Sea } \\
\text { region }\end{array}$ & & $\begin{array}{l}\text { Flavobacterium } \\
\text { psychrophilum }^{b} / \\
\text { aquidurense }^{a}\end{array}$ \\
\hline P169 & $\begin{array}{l}\text { Oncorhynchus } \\
\text { mykiss }\end{array}$ & egg & 2017 & January & Çağlayan & Rize & $\begin{array}{l}\text { Black Sea } \\
\text { region }\end{array}$ & & Flavobacterium hydatis ${ }^{a}$ \\
\hline V3 & DSM 7189 & soil & 1992 & July & California & $\mathrm{ABD}$ & & & \\
\hline V8 & DSM 50090 & $\begin{array}{l}\text { pre-filter } \\
\text { tanks }\end{array}$ & 1990 & August & $\begin{array}{l}\text { Northern } \\
\text { Ireland }\end{array}$ & $\begin{array}{l}\text { United } \\
\text { Kingdom }\end{array}$ & & & \\
\hline V34 & $\begin{array}{l}\text { Oncorhynchus } \\
\text { mykiss }\end{array}$ & 200 & 2013 & July & Uzunyayla & Kayseri & $\begin{array}{l}\text { Central } \\
\text { Anatolia }\end{array}$ & $\begin{array}{l}\text { Darkening in color and } \\
\text { exopthalmia }\end{array}$ & \\
\hline V35 & $\begin{array}{l}\text { Oncorhynchus } \\
\text { mykiss }\end{array}$ & 200 & 2013 & July & Uzunyayla & Kayseri & $\begin{array}{l}\text { Central } \\
\text { Anatolia }\end{array}$ & $\begin{array}{l}\text { Darkening in color and } \\
\text { exopthalmia }\end{array}$ & \\
\hline V36 & $\begin{array}{l}\text { Oncorhynchus } \\
\text { mykiss }\end{array}$ & 200 & 2013 & July & Uzunyayla & Kayseri & $\begin{array}{l}\text { Central } \\
\text { Anatolia }\end{array}$ & $\begin{array}{l}\text { Darkening in color and } \\
\text { exopthalmia }\end{array}$ & \\
\hline V37 & $\begin{array}{l}\text { Oncorhynchus } \\
\text { mykiss }\end{array}$ & 200 & 2013 & July & Uzunyayla & Kayseri & $\begin{array}{l}\text { Central } \\
\text { Anatolia }\end{array}$ & $\begin{array}{l}\text { Darkening in color and } \\
\text { exopthalmia }\end{array}$ & \\
\hline V38 & $\begin{array}{l}\text { Oncorhynchus } \\
\text { mykiss }\end{array}$ & 200 & 2013 & July & Uzunyayla & Kayseri & $\begin{array}{l}\text { Central } \\
\text { Anatolia }\end{array}$ & $\begin{array}{l}\text { Darkening in color and } \\
\text { exopthalmia }\end{array}$ & \\
\hline V42 & $\begin{array}{l}\text { Oncorhynchus } \\
\text { mykiss }\end{array}$ & 200 & 2013 & June & Su Şehri & Sivas & $\begin{array}{l}\text { Central } \\
\text { Anatolia }\end{array}$ & $\begin{array}{l}\text { Darkening in color and } \\
\text { exopthalmia }\end{array}$ & $\begin{array}{l}\text { Aeromonas media } \\
(\mathrm{MG} 310171)^{\mathrm{c}}\end{array}$ \\
\hline V43 & $\begin{array}{l}\text { Oncorhynchus } \\
\text { mykiss }\end{array}$ & 200 & 2013 & June & Su Şehri & Sivas & $\begin{array}{l}\text { Central } \\
\text { Anatolia }\end{array}$ & $\begin{array}{l}\text { Darkening in color and } \\
\text { exopthalmia }\end{array}$ & $\begin{array}{l}\text { Aeromonas media } \\
(\mathrm{MG} 310171)^{\mathrm{c}}\end{array}$ \\
\hline V45 & $\begin{array}{l}\text { Oncorhynchus } \\
\text { mykiss }\end{array}$ & 200 & 2013 & June & Su Şehri & Sivas & $\begin{array}{l}\text { Central } \\
\text { Anatolia }\end{array}$ & $\begin{array}{l}\text { Darkening in color and } \\
\text { exopthalmia }\end{array}$ & $\begin{array}{l}\text { Aeromonas media } \\
(\mathrm{MG} 310171)^{\mathrm{c}}\end{array}$ \\
\hline V81 & $\begin{array}{l}\text { Oncorhynchus } \\
\text { mykiss }\end{array}$ & 200 & 2013 & July & Su Şehri & Sivas & $\begin{array}{l}\text { Central } \\
\text { Anatolia }\end{array}$ & $\begin{array}{l}\text { Darkening in color and } \\
\text { exopthalmia }\end{array}$ & $\begin{array}{l}\text { Aeromonas media } \\
(\mathrm{MG} 310171)^{\mathrm{c}}\end{array}$ \\
\hline
\end{tabular}

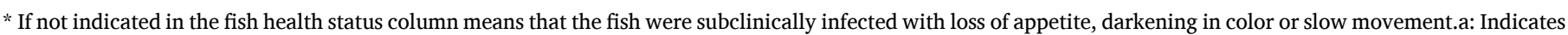

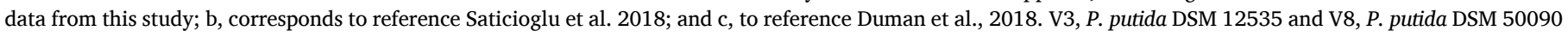
are bacterial controls.

calculated according to the intensity of bacterial fluorescence, ranging from poor $(+)$ to very rich $(++++)$, and compared among the bacteria by UV transillumination as described by Lamichhane and Varvaro (2013). The reference strains Pseudomonas fluorescens DSM 50090 and Pseudomonas putida DSM 12735 were used as controls to validate the biochemical tests.

The bacteria were also tested for survival in sterile distilled water and sterile tap water alone. Distilled water (conductivity, $3 \mathrm{mS} / \mathrm{cm}$ ) and tap water (conductivity, $343 \mathrm{mS} / \mathrm{cm}$ ) were sterilized in an autoclave and distributed into 24 well-plates. Bacterial suspensions prepared from pure and fresh cultures were adjusted to 0.5 McFarland turbidity, and $100-\mu l$ aliquots of the bacterial suspensions were added to the plate wells. After the plates were incubated at $28{ }^{\circ} \mathrm{C}$ for 24 and $48 \mathrm{~h}$, optical density was measured at a wavelength of $595 \mathrm{~nm}$ using a microplate reader (Multiskan Go, Thermo Fisher Scientific, Waltham, MA, USA), and survival was defined in comparison to the control sample (without bacteria) (CLSI, 2017).

\subsection{Antibiotic resistance}

Resistance to antibiotics of different groups was determined using the disc diffusion method by complying with the performance standards for the antimicrobial susceptibility testing of bacteria isolated from aquatic animals (Clinical and Laboratory Standards Institute, CLSI guidelines M42-A) (CLSI - Clinical and Laboratory Standards Institute,
2006). The antibiotics used in the susceptibility tests included sulfonamides (trimethoprim/sulfamethoxazole, SXT, 1/19), aminopenicillins (amoxicillin, AML), tetracyclines (doxycycline, DO; tetracycline, TE; oxytetracycline, OT), fluoroquinolones (enrofloxacin, ENR; ciprofloxacin, CIP; flumequine, UB), aminoglycosides (gentamicin, $\mathrm{CN}$ ), penicillins (ampicillin, AMP), macrolides (erythromycin, E), clindamycin (lincomycin, MY), and chloramphenicol (florfenicol, FFC). Antibiotic discs were placed on Mueller-Hinton agar (MHA) plates previously inoculated with a pure culture of the strains. After incubation at $28{ }^{\circ} \mathrm{C}$ (24-28 h), inhibition zones were measured in $\mathrm{mm}$ and compared with the critical values of the European Committee on Antimicrobial Susceptibility Testing (EUCAST-European Committee on Antimicrobial Susceptibility Testing, 2020, www.eucast.org) to evaluate whether a strain was sensitive or resistant. E. coli ATCC 25922 was used as the quality control (QC) strain, and the acceptable ranges set by the CLSI for this strain were applied (CLSI - Clinical and Laboratory Standards Institute, 2014).

\subsection{DNA extraction, PCR amplification, and DNA sequencing conditions}

The genomic DNA of the bacterial isolates was extracted using a QIAamp DNA mini kit (Qiagen, Hilden, Germany) according to the manufacturer's instructions. All DNA samples were optimized according to concentration and purity before molecular analysis. PCR amplification, primer selection, purification of the amplified products, DNA 
sequencing, and the sequence analysis procedures were performed according to previous descriptions (Mulet et al., 2017).

\subsection{Phylogenetic analysis}

Individual trees were generated from the isolates, and multilocus sequence analyses (MLSA) were carried out based on the concatenated partial sequences of the 16S rRNA (1263 nt), gyrB (RNA gyrase subunit B, $810 \mathrm{nt}$ ), rpoD (RNA polymerase subunit D, $694 \mathrm{nt}$ ), and rpoB (RNA polymerase beta subunit, $923 \mathrm{nt}$ ) genes, totalling $3690 \mathrm{nt}$ (Mulet et al., 2017). The EZ Bio Cloud Database, https://www.ezbiocloud.net/, was used to affiliate the 16S rRNA sequences to the genus Pseudomonas (Yoon et al., 2017). The Jukes-Cantor (JC), maximum likelihood (ML) (Jukes and Cantor, 1969; Felsenstein, 1981), and maximum parsimony (MP) (Nei and Kumar, 2000) algorithms were used for comparisons.

\subsection{Statistical analysis}

Analyses of variance, standard deviation calculations and regression analyses were performed using the Big Data analysis program with conventional standard deviation analysis (Analytics, 2013). Multiple regression analysis was used to analyse the results in relation to Pseudomonas species count and species score, the number of cases associated with the organ of isolation, water temperature, fish length, month of isolation and mixed isolation status. Graphics of the statistical results were constructed with Excel from the Tableau database (Analytics, 2013). Isolates, for which information was incomplete (i.e. isolation date, water temperature), were not included in the statistical analysis.

\subsection{Ethical statements}

This research was supported by the Scientific and Technological
Research Council of Turkey and approved by the Local Ethics Commission (report 2012-14-04).

\section{Results}

\subsection{Bacterial isolation}

A total of 90 Pseudomonas strains were isolated, including 81 from rainbow trouts (Onchorhynchus mykiss, OM), three from pond water, three from brook trouts (Salvelinus fontinalis, SF), two from brown trouts (Salmo trutta macrostigma, STM) and one from the Black sea trout (Salmo trutta labrax, STL). Origins varied from egg to brood stock fish $(3000 \mathrm{~g})$ or yolk-sac and pond water. Most strains were recovered from fish of a weight below $10 \mathrm{~g}$ (Table 1, supplementary Fig. S1). Thirty-eight percent of the fish presented with disease symptoms or lesions, such as anaemia, darkening in colour, dorsal fin erosion, exophthalmia, high mortality, loss of appetite, and abnormal swimming, and some fish were moribund as indicated in Table 1. Pseudomonas spp. were isolated in four different regions of Turkey and were detected at higher rates in the Central Anatolia and Aegean regions. No isolates were recovered from the samples taken in the Marmara and Mediterranean regions. The distribution of the isolates is presented in Fig. 1 (and in the following interactive link together with GPS data and information on farm location, Pseudomonas species, town, province and region https://www. google.com/maps/d/viewer?hl=en\&mid=11E1b5mFWsZeKwNwpA wj4ItdO3FUz2rWd\&11=39.54525767883057\%2C35.11440467765806 $\& \mathrm{z}=5$ ). The numbers of the Pseudomonas species identified and their distribution by provinces are shown in Fig. 2 .

In 56 fish without any symptoms, 23 Flavobacterium strains were isolated together with Pseudomonas strains. The Flavobacterium species identified include $F$. aquidurense, $F$. branchiarum, $F$. collinsii, $F$. frigidimaris, F. glaciei, F. hydatis, F. oncorhynchi, F. piscis, F. psychrophilum,

\section{P. haemolytica \\ P Pseudomonas sp. (D) \\ - P. meridiana \\ ○ P. defensor \\ - P. mandelii \\ (3) Pseudomonas sp. (B) \\ - P. weihenstephanensis \\ $\diamond$ Pseudomonas sp. (C) \\ Pseudomonas sp. (E) \\ P. brenneri \\ (C) P.lactis \\ P. proteolytica \\ ( Pseudomonas sp. (F) \\ (- Pseudomonas sp. (A) \\ $\square$ P. laurylsulfatovorans \\ - P. lundensis \\ O P. lurida \\ 조. migulae \\ O P. simiae}

\ Pseudomonas sp. (G)

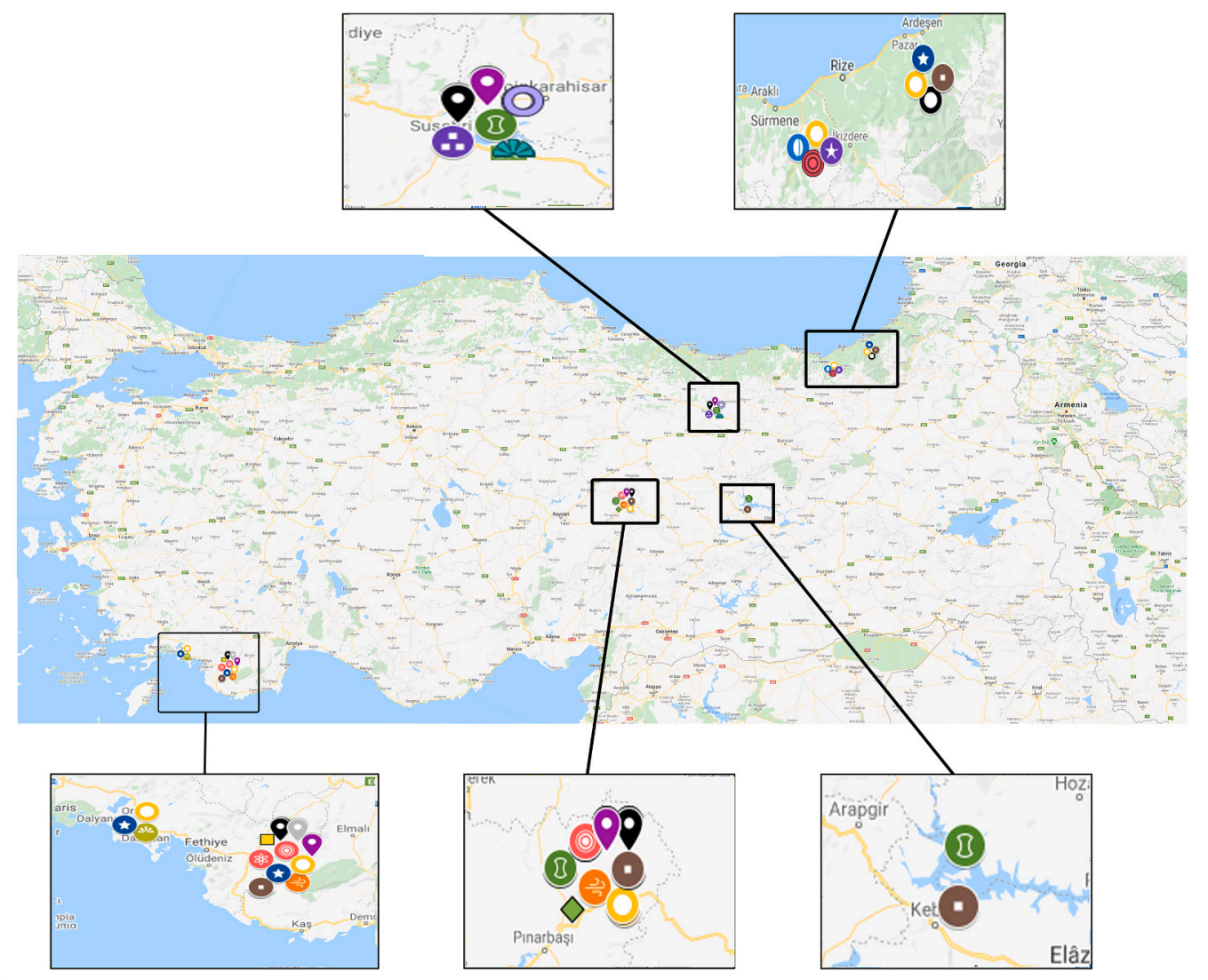

Fig. 1. Map showing the distribution of Pseudomonas species in fish farms in Turkey. 


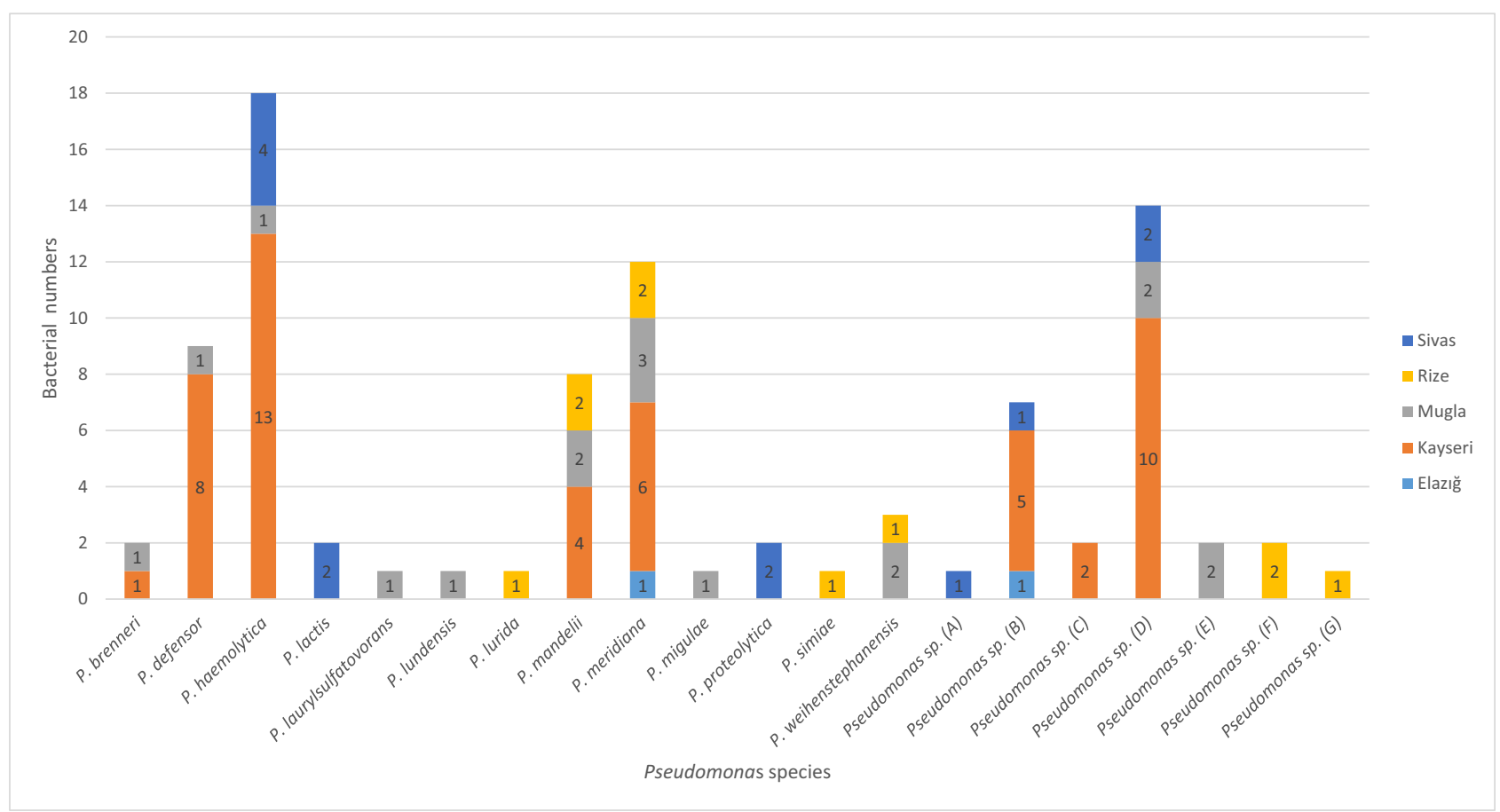

Fig. 2. Distribution and number of Pseudomonas species in fish farms in Turkey.

F. tiangeerense and F. tructae. In six cases, more than one species of Flavobacterium were recovered. From the samples taken from diseased fish, apart from 34 Pseudomonas strains, 8 strains of motile Aeromonas and Flavobacterium were recovered and identified as Aeromonas media, F. collinsii, and Flavobacterium spp. (Table 1).

\subsection{Phylogenetic identification}

The phylogenetic assignation of the Pseudomonas strains at species level is summarized in Fig. 3. An initial analysis of the 16S rRNA sequences affiliated the isolates to the genus Pseudomonas, as shown in Supplementary Table 1, but the low species differentiation power of this gene did not allow identification at species level. Eighty-eight of the 90 strains belonged to the Pseudomonas fluorescens group and its six subgroups: $P$. fluorescens SG (39 strains), P. gessardii SG (16 strains), $P$. fragi SG (4 strains), $P$. mandellii SG (13 strains), $P$. koreensis SG (15 strains) and $P$. jessenii SG (1 strain). Only two strains were classified under the $P$. putida group (Pseudomonas sp. E). For a precise identification at species level, the $r p o D$ gene sequences were analysed for all strains. At a cutoff value of 95\%, 61 strains were identified at species level and affiliated to 14 different Pseudomonas species, the most prevalent of which were P. haemolytica (18 strains), P. meridiana (12 strains), P. defensor (9 strains) and $P$. mandelii ( 8 strains). The remaining species were represented by 1 to 3 strains, as shown in Table 2, supplementary Table 1, and Fig. 3, and are detailed in supplementary Figs. 2 and 3.

To further phylogenetically characterize the remaining 29 strains (32\%), a MLSA based on the concatenated sequences of the gyrB, rpoB, $r p o D$ and 16S rRNA genes was performed. The results are shown in Fig. 3, supplementary Fig. 3 and supplementary Table 1. The cut-off value to assign a strain to a species is $97 \%$ in the 4-gene MLSA. Seven presumptive new species, previously not described, could be delineated and were labelled with capital letters as follows: Pseudomonas sp. (A), 1 strain; Pseudomonas sp. (B), 7 strains; Pseudomonas sp. (C), 2 strains; Pseudomonas sp. (D), 14 strains; Pseudomonas sp. (E), 2 strains; Pseudomonas sp. (F), 2 strains and Pseudomonas sp. (G), 1 strain (supplementary Table 1). Pseudomonas sp. (F) and Pseudomonas sp. (G) were $97.1 \%$ similar to known species type strains, but could not be assigned to any of them in the phylogenetic tree. Pseudomonas sp. (B) and (D) were isolated most frequently in the Kayseri province, and P. meridiana and Pseudomonas sp. (B) were isolated in Elazig. Supplementary Table 2 shows the GenBank nucleotide accession numbers of the sequences used in this study. The accession numbers indicated in bold are for the sequences determined in this study.

\subsection{Physiological and biochemical characterization}

All strains shared the essential phenotypic characteristics of members of the genus Pseudomonas. They were Gram-negative, motile, rodshaped, oxidase- and catalase-positive, non-fermentative bacteria with a strictly oxidative metabolism, and $84 \%$ of them produced diffusible fluorescent pigments. These were the primary strain selection criteria for this study, and the 90 strains underwent detailed phenotypic characterization to assess their fitness and probable role in the aquaculture setting. Results are shown in Table 2 . All strains were able to grow at $\mathrm{NaCl}$ concentrations of $0-4.5 \%$, but most of them did not tolerate higher concentrations of $\mathrm{NaCl}$, such that $20 \%$ of the strains did not grow on $6 \%$ $\mathrm{NaCl}, 61 \%$ did not grow on $7 \% \mathrm{NaCl}, 73 \%$ did not grow on $8 \% \mathrm{NaCl}$ and $91 \%$ did not grow on $9 \% \mathrm{NaCl}$. The only species that tolerated $9 \% \mathrm{NaCl}$ were P. haemolytica (P45, P91 and P93), P. lundensis (P131), $P$. proteolytica (P102), P. weihenstephanensis (P132), and Pseudomonas sp. (D) (P10 and P42).

In order to determine the potential environmental and public health risks posed by the isolates, their survival in sterile distilled water and tap water was tested, and all isolates were observed to survive throughout the test period. All of the isolates grew at $4{ }^{\circ} \mathrm{C}, 25^{\circ} \mathrm{C}$, and $37^{\circ} \mathrm{C}$ with the exception of $P$. defensor (P57) and Pseudomonas sp. (C) (P50), which did not grow at $4{ }^{\circ} \mathrm{C}$, and P. defensor (P101), P. haemolytica (P16, P95), P. mandelii (P5), P. proteolytica (P102), Pseudomonas sp. (B) (P29), and Pseudomonas sp. (D) (P25), which did not grow at $37{ }^{\circ} \mathrm{C}$. P. haemolytica (V81) displayed the highest growth temperature, which was $45^{\circ} \mathrm{C}$. As indicated in Table 2, some strains produced a pellicle in the liquid-air interphase under different salinity and temperature conditions. The analysis of the proteolytic activity of the isolates demonstrated that $91 \%$ tested positive for this activity, as shown in Table 2. Haemolytic activity was detected in $P$. haemolytica, $P$. lactis, $P$. lurida, $P$. meridiana (only P167, P168, V37 and V38), Pseudomonas sp. (B) (excluding P7), 


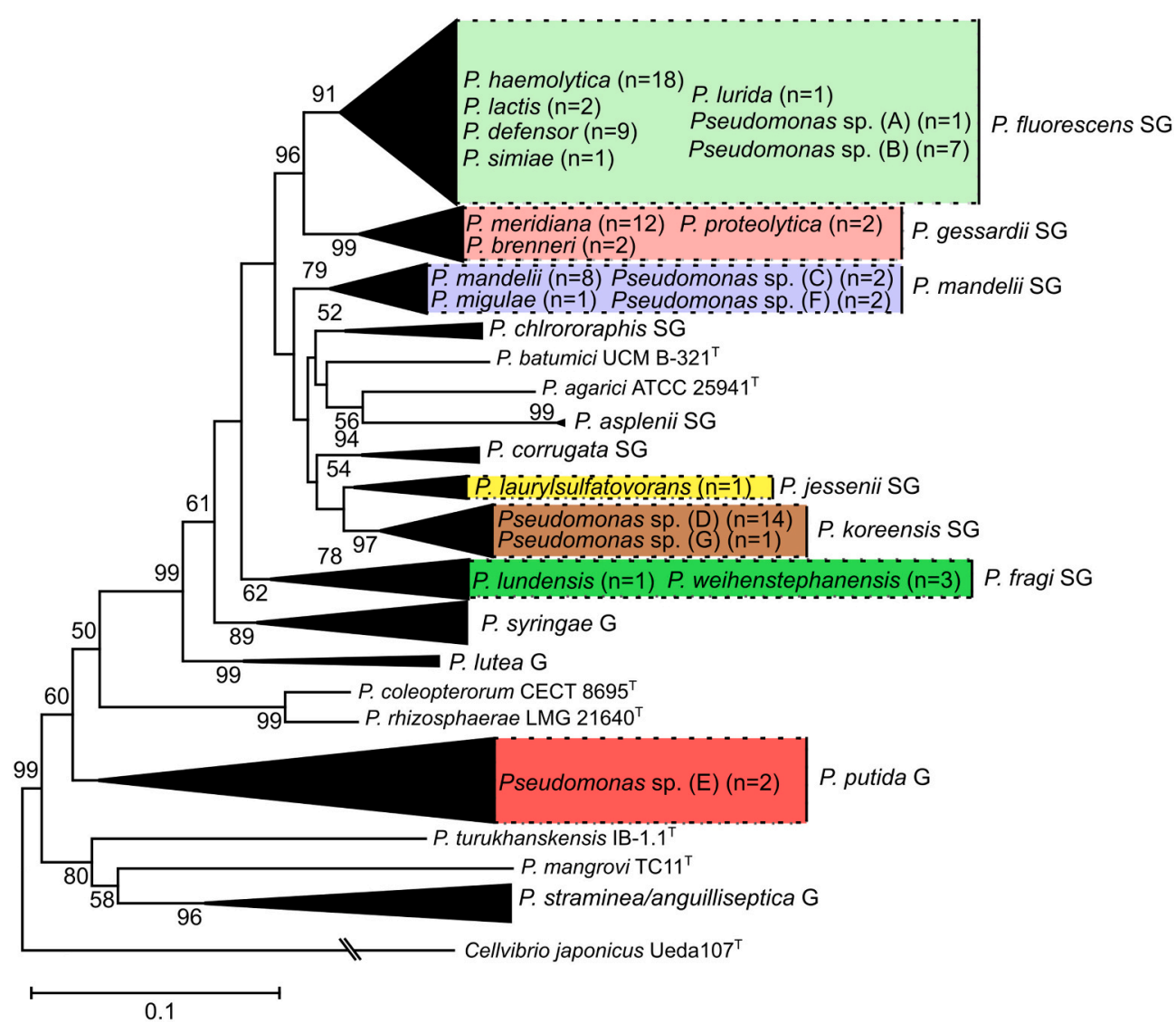

Fig. 3. Phylogenetic tree of the strains isolated in this study and Pseudomonas species type strains of the Pseudomonas fluorescens lineage, based on the partial sequences of the rpoD gene. Isolates from this study are highlighted in colour and " $\mathrm{n}$ " indicates the number of strains. Cellvibrio japonicus Ueda107 was used as an outgroup. The bar indicates sequence divergence. Bootstrap values higher than $50 \%$ (from 1000 replicates) are indicated at the nodes.

Pseudomonas sp. (C), Pseudomonas sp. (D) (P1, P24-1, P42, and P141), and Pseudomonas sp. (G). The remaining strains were non-haemolytic.

\subsection{Antibiotic susceptibility}

Antibiotic breakpoints are not available in the CLSI database for the genus Pseudomonas, with the exception of $P$. aeruginosa. The bacterial isolates were evaluated for their antibiotic sensitivity to nine different groups of antibiotics, but only ciprofloxacin (CIP) susceptibility values were given in the EUCAST updated breakpoint table (EUCAST-European Committee on Antimicrobial Susceptibility Testing, Version 10.0, valid from 2020 to 01-01). According to EUCAST data (2020), Pseudomonas spp. are susceptible if the breakpoint is higher than $50 \mathrm{~mm}$, and isolates are assumed to be intermediately resistant when breakpoints are between 50 and $26 \mathrm{~mm}$. Accordingly, while $P$. lurida and Pseudomonas sp. (E) were determined to be resistant $(<26 \mathrm{~mm})$ to ciprofloxacin, the remaining isolates were assumed to be intermediately resistant. All isolates were fully resistant to lincomycin (MY), and the majority were resistant to trimethoprim/sulfamethoxazole (SXT, 1/19), amoxicillin (AML), ampicillin (AMP), erythromycin (E), and florfenicol (FFC) with no growth zone $(0 \mathrm{~mm})$ in the disc diffusion test. Most Pseudomonas species showed an inhibition zone for doxycycline (DO), tetracycline (TE), oxytetracycline (OT), enrofloxacin (ENR), ciprofloxacin (CIP), flumequine (UB), and gentamicin (CN), but were not evaluated for antibiotic susceptibility or resistance. Pseudomonas sp. (D), (E), (F), $P$. defensor, and $P$. meridiana were the most resistant species among all identified Pseudomonas with no inhibition zone $(0 \mathrm{~mm})$ as indicated in supplementary Table S3.

\subsection{Statistical results}

All data were compared by using logistic regression analysis tests and were analysed by an iteratively reweighted least squares methodology. The number of strains isolated from healthy fish was 56, while 34 species were detected in fish displaying various symptoms. The number of accompanying species was 31, and in 6 asymptomatic cases, more than one species was isolated in association with Pseudomonas spp. The species most frequently isolated from rainbow trouts were $P$. haemolytica, Pseudomonas sp. (D), P. meridiana, P. defensor and P. mandelii (Fig. 1, http $\mathrm{s}: / /$ www.google.com/maps/d/viewer?hl=en\&mid=1lE1b5mFWsZe KwNwpAwj4ItdO3FUz2rWd\&1l=39.54525767883057\%2C35.1144046 $7765806 \& \mathrm{z}=5$, and Fig. 2). All species, excluding P. simiae and P. lurida, were isolated from rainbow trouts, while Pseudomonas sp. (B) was the only one recovered from pond water (supplementary Fig. S4). Pseudomonas species were isolated from different regions of Turkey, more frequently from Kayseri, Mugla and Sivas, and less frequently from Rize and Elazig (Figs. 1 and 2). Statistical analyses demonstrated a correlation between species and the month of isolation. P. haemolytica was frequently isolated in July, $P$. meridiana in January, and Pseudomonas sp. (D) in December. None of the Pseudomonas spp. were isolated in May, September and October. A heterogenic species distribution was detected during the 2010-2015 and 2015-2020 periods. After 2015, Pseudomonas were frequently detected in January, April and September (supplementary Fig. S5). Ten species were isolated from injured fish with symptoms, such as anaemia, darkening in colour, fin erosion, exophthalmia and abnormal swimming. These symptoms were mainly attributed to a possible infection with $P$. haemolytica, $P$. meridiana and $P$. defensor (Fig. 4, Table 1). P. migulae presented the highest growth temperature, and $P$. proteolytica presented with the lowest growth 
Table 2

The identification, and physiological and biochemical characteristics of the strains.

\begin{tabular}{|c|c|c|c|c|c|c|c|c|c|c|c|c|c|c|c|c|c|c|c|}
\hline \multirow[t]{2}{*}{ Strain } & \multirow[t]{2}{*}{ Bacteria } & \multirow[t]{2}{*}{ Fluorescence } & \multirow{2}{*}{$\begin{array}{l}\text { Pellicle } \\
\text { formation }\end{array}$} & \multirow{2}{*}{$\begin{array}{l}\text { Haemolysis on } \\
\text { blood agar }\end{array}$} & \multirow[t]{2}{*}{ Motility } & \multirow[b]{2}{*}{ Oxidase } & \multicolumn{5}{|c|}{ Growth with $\mathrm{NaCl}(\%)$} & \multicolumn{2}{|l|}{ Survival } & \multicolumn{5}{|c|}{ Growth temperature $\left({ }^{\circ} \mathrm{C}\right)$} & \multirow{2}{*}{$\begin{array}{l}\text { Proteolytic } \\
\text { activity }\end{array}$} \\
\hline & & & & & & & $\begin{array}{l}\mathrm{b}_{0-} \\
4.5\end{array}$ & 6 & 7 & 8 & $\overline{9}$ & $\begin{array}{l}\text { Destilled water } \\
(3 \mu \mathrm{m})\end{array}$ & $\begin{array}{l}\text { Tap water } \\
(343 \mu \mathrm{m})\end{array}$ & 4 & 25 & 37 & 42 & $\overline{45}$ & \\
\hline P34 & P. brenneri & - & - & - & + & + & + & + & - & - & - & + & +++ & \pm & + & + & - & - & + \\
\hline P114 & P. brenneri & +++ & - & - & + & + & + & - & - & - & - & ++ & ++++ & + & + & + & - & - & + \\
\hline P21 & P. defensor & - & + & - & + & + & + & + & - & - & - & ++ & +++ & + & + & + & - & - & + \\
\hline P47 & P. defensor & +++ & + & - & + & + & + & + & + & + & - & ++ & ++ & + & + & + & - & - & + \\
\hline P54 & P. defensor & ++ & + & - & + & + & + & + & - & - & - & ++ & +++ & + & + & + & - & - & + \\
\hline P55 & P. defensor & - & + & - & + & + & + & + & - & - & - & ++ & +++ & + & + & + & - & - & + \\
\hline P57 & P. defensor & ++ & - & - & + & + & + & + & + & + & - & ++ & +++ & - & + & + & - & - & + \\
\hline P58 & P. defensor & + & + & - & + & + & + & + & - & - & - & ++ & +++ & + & + & + & - & - & + \\
\hline P99 & P. defensor & +++ & - & - & + & + & + & + & + & + & - & ++ & ++++ & + & + & + & - & - & + \\
\hline P100 & P. defensor & +++ & + & - & + & + & + & + & + & + & - & ++ & ++++ & + & + & + & - & - & + \\
\hline P101 & P. defensor & ++ & + & - & + & + & + & + & + & + & - & ++ & ++++ & + & + & - & - & - & + \\
\hline P16 & P. haemolytica & + & - & - & + & + & + & + & - & - & - & ++ & +++ & + & + & - & - & - & + \\
\hline P43 & P. haemolytica & ++++ & + & $\beta$ & + & + & + & + & - & - & - & ++ & +++ & + & + & + & - & - & + \\
\hline P44 & P. haemolytica & +++ & - & $\beta$ & + & + & + & + & + & + & - & ++ & +++ & + & + & + & - & - & + \\
\hline P45 & P. haemolytica & +++ & + & $\beta$ & + & + & + & + & + & + & + & ++ & +++ & + & + & + & - & - & + \\
\hline P46 & P. haemolytica & +++ & + & $\beta$ & + & + & + & + & + & + & - & ++ & +++ & + & + & + & - & - & + \\
\hline P52 & P. haemolytica & ++++ & + & $\beta$ & + & + & + & + & - & - & - & ++ & +++ & + & + & + & - & - & + \\
\hline P56 & P. haemolytica & + & + & $\beta$ & + & + & + & + & - & - & - & ++ & +++ & + & + & + & - & - & + \\
\hline P59 & P. haemolytica & ++++ & - & $\beta$ & + & + & + & + & - & - & - & ++ & +++ & + & + & + & - & - & + \\
\hline P68b & P. haemolytica & ++ & + & $\beta$ & + & + & + & + & - & - & - & ++ & +++ & + & + & + & - & - & + \\
\hline P91 & P. haemolytica & +++ & + & $\beta$ & + & + & + & + & + & + & + & ++ & +++ & + & + & + & - & - & + \\
\hline P93 & P. haemolytica & +++ & + & $\beta$ & + & + & + & + & + & + & + & ++ & ++++ & + & + & + & - & - & + \\
\hline P94 & P. haemolytica & +++ & + & $\beta$ & + & + & + & + & + & - & - & ++ & ++++ & + & + & + & - & - & + \\
\hline P95 & P. haemolytica & +++ & + & $\beta$ & + & + & + & + & + & + & - & ++ & ++++ & + & + & - & - & - & + \\
\hline P97 & P. haemolytica & +++ & + & $\beta$ & + & + & + & + & - & - & - & ++ & ++++ & + & + & + & - & - & + \\
\hline V42 & P. haemolytica & + & + & $\beta$ & + & + & + & + & + & - & - & ++ & +++ & + & + & + & - & - & + \\
\hline V43 & P. haemolytica & + & + & $\beta$ & + & + & + & + & + & - & - & ++ & +++ & + & + & + & - & - & + \\
\hline V45 & P. haemolytica & + & + & $\beta$ & + & + & + & + & + & - & - & ++ & +++ & + & + & + & - & - & + \\
\hline V81 & P. haemolytica & ++ & + & $\beta$ & + & + & + & + & + & - & - & ++ & +++ & + & + & + & + & + & + \\
\hline P48 & P. lactis & +++ & + & $\beta$ & + & + & + & + & + & + & - & ++ & +++ & + & + & + & - & - & + \\
\hline P106 & P. lactis & +++ & + & $\beta$ & + & + & + & + & + & + & - & ++ & ++++ & + & + & + & - & - & + \\
\hline P138 & P. laurylsulfatovorans & - & - & - & + & + & + & - & - & - & - & ++ & +++ & + & + & + & - & - & + \\
\hline P131 & P. lundensis & - & - & - & + & + & + & + & + & + & + & ++ & +++ & + & + & + & - & - & + \\
\hline P163 & P. lurida & ++++ & - & $\beta$ & + & + & + & - & - & - & - & ++ & +++ & + & + & + & - & - & + \\
\hline Р3a & P. mandelii & ++ & - & - & + & + & + & + & - & - & - & ++ & +++ & + & + & + & - & - & + \\
\hline P3b & P. mandelii & ++ & - & - & + & + & + & + & - & - & - & ++ & +++ & + & + & + & - & - & + \\
\hline P5 & P. mandelii & - & - & - & + & + & + & + & - & - & - & ++ & +++ & + & + & - & - & - & + \\
\hline P31 & P. mandelii & + & - & - & + & + & + & + & - & - & - & ++ & +++ & \pm & + & + & + & - & + \\
\hline P124 & P. mandelii & - & - & - & + & + & + & - & - & - & - & ++ & +++ & + & + & + & - & - & + \\
\hline P135 & P. mandelii & - & - & - & + & + & + & - & - & - & - & ++ & +++ & + & + & + & - & - & - \\
\hline P164 & P. mandelii & - & - & & + & + & + & - & - & - & - & ++ & +++ & + & + & + & - & - & + \\
\hline P169 & P. mandelii & - & - & - & + & + & + & - & - & - & - & ++ & +++ & + & + & + & - & - & + \\
\hline P121 & P. meridiana & ++++ & - & - & + & + & + & + & + & + & - & ++ & +++ & + & + & + & - & - & + \\
\hline P123 & P. meridiana & - & - & - & + & + & + & + & + & - & - & ++ & +++ & + & + & + & - & - & + \\
\hline P137 & P. meridiana & ++++ & - & - & + & + & + & + & - & - & - & ++ & +++ & + & + & + & - & - & + \\
\hline P147 & P. meridiana & ++++ & - & - & + & + & + & + & - & - & - & ++ & ++++ & + & + & + & - & - & + \\
\hline P162 & P. meridiana & ++ & - & - & + & + & + & - & - & - & - & ++ & +++ & + & + & + & - & - & + \\
\hline P167 & P. meridiana & - & - & $\beta$ & + & + & + & - & - & - & - & ++ & +++ & + & + & + & - & - & + \\
\hline P168 & P. meridiana & +++ & - & $\beta$ & + & + & + & - & - & - & - & ++ & +++ & + & + & + & - & - & + \\
\hline V34 & P. meridiana & ++ & + & - & + & + & + & + & + & - & - & ++ & ++++ & + & + & + & - & - & + \\
\hline V35 & P. meridiana & ++ & + & - & + & + & + & + & + & - & - & ++ & ++++ & + & + & + & + & - & + \\
\hline
\end{tabular}


Table 2 (continued)

\begin{tabular}{|c|c|c|c|c|c|c|c|c|c|c|c|c|c|c|c|c|c|c|c|}
\hline \multirow[t]{2}{*}{ Strain } & \multirow[t]{2}{*}{ Bacteria } & \multirow[t]{2}{*}{ Fluorescence } & \multirow{2}{*}{$\begin{array}{l}\text { Pellicle } \\
\text { formation }\end{array}$} & \multirow{2}{*}{$\begin{array}{l}\text { Haemolysis on } \\
\text { blood agar }\end{array}$} & \multirow[t]{2}{*}{ Motility } & \multirow[b]{2}{*}{ Oxidase } & \multicolumn{5}{|c|}{ Growth with $\mathrm{NaCl}$ (\%) } & \multicolumn{2}{|l|}{ Survival } & \multicolumn{5}{|c|}{ Growth temperature $\left({ }^{\circ} \mathrm{C}\right)$} & \multirow{2}{*}{$\begin{array}{l}\text { Proteolytic } \\
\text { activity }\end{array}$} \\
\hline & & & & & & & $\begin{array}{l}\mathrm{b}_{0-} \\
4.5\end{array}$ & 6 & 7 & 8 & 9 & $\begin{array}{l}\text { Destilled water } \\
(3 \mu \mathrm{m})\end{array}$ & $\begin{array}{l}\text { Tap water } \\
(343 \mu \mathrm{m})\end{array}$ & 4 & 25 & 37 & 42 & 45 & \\
\hline V36 & P. meridiana & ++ & + & - & + & + & + & + & + & - & - & ++ & ++++ & + & + & + & + & - & + \\
\hline V37 & P. meridiana & ++ & + & $\beta$ & + & + & + & + & - & - & - & ++ & ++++ & + & + & + & - & - & + \\
\hline V38 & P. meridiana & ++ & + & $\beta$ & + & + & + & + & - & - & - & ++ & +++ & + & + & + & - & - & + \\
\hline P109 & P. migulae & +++ & - & - & + & + & + & - & - & - & - & ++ & +++ & + & + & + & + & - & + \\
\hline P51 & P. proteolytica & ++++ & + & - & + & + & + & + & - & - & - & ++ & +++ & + & + & + & - & - & + \\
\hline P102 & P. proteolytica & ++ & + & - & + & + & + & + & + & + & + & ++ & +++ & + & + & - & - & - & + \\
\hline P156 & P. simiae & ++++ & - & - & + & + & + & - & - & - & - & ++ & +++ & + & + & + & - & - & + \\
\hline P122 & P. weihenstephanensis & - & - & - & + & + & + & + & - & - & - & ++ & +++ & + & + & + & - & - & - \\
\hline P132 & P. weihenstephanensis & ++ & - & - & + & + & + & + & + & + & + & ++ & +++ & + & + & + & - & - & + \\
\hline P166 & P. weihenstephanensis & - & - & - & + & + & + & - & - & - & - & ++ & +++ & \pm & + & + & - & - & + \\
\hline P115 & Pseudomonas sp. (A) & ++++ & - & - & + & + & + & + & - & - & - & + & +++ & + & + & + & - & - & + \\
\hline P7 & Pseudomonas sp. (B) & ++ & - & - & + & + & + & + & - & - & - & ++ & +++ & + & + & + & - & - & + \\
\hline P11 & Pseudomonas sp. (B) & ++++ & - & $\beta$ & + & + & + & + & - & - & - & ++ & +++ & + & + & + & - & - & + \\
\hline P24-2 & Pseudomonas sp. (B) & ++++ & - & $\beta$ & + & + & + & + & - & - & - & ++ & +++ & \pm & + & + & + & - & + \\
\hline P29 & Pseudomonas sp. (B) & +++ & - & $\beta$ & + & + & + & + & - & - & - & ++ & +++ & + & + & - & - & - & + \\
\hline P72 & Pseudomonas sp. (B) & +++ & + & $\beta$ & + & + & + & + & + & + & - & ++ & ++++ & + & + & + & - & - & + \\
\hline P73 & Pseudomonas sp. (B) & +++ & - & $\beta$ & + & + & + & + & + & + & - & ++ & ++++ & + & + & + & - & - & + \\
\hline P158 & Pseudomonas sp. (B) & ++++ & - & $\beta$ & + & + & + & - & - & - & - & ++ & +++ & + & + & + & - & - & + \\
\hline P49 & Pseudomonas sp. (C) & +++ & - & $\alpha$ & + & + & + & - & - & - & - & ++ & ++++ & + & + & + & - & - & - \\
\hline P50 & Pseudomonas sp. (C) & + & - & $\alpha$ & + & + & + & + & - & - & - & ++ & +++ & - & + & + & - & - & - \\
\hline P1 & Pseudomonas sp. (D) & + & - & $\alpha$ & + & + & + & + & - & - & - & ++ & +++ & + & + & + & - & - & + \\
\hline P2 & Pseudomonas sp. (D) & + & - & - & + & + & + & + & - & - & - & ++ & +++ & + & + & + & - & - & + \\
\hline P9 & Pseudomonas sp. (D) & ++++ & - & - & + & + & + & + & + & + & - & ++ & +++ & + & + & + & - & - & + \\
\hline P10 & Pseudomonas sp. (D) & + & - & - & + & + & + & + & + & + & + & ++ & +++ & + & + & + & - & - & + \\
\hline P24-1 & Pseudomonas sp. (D) & + & - & $\alpha$ & + & + & + & + & - & - & - & ++ & +++ & + & + & + & - & - & + \\
\hline P25 & Pseudomonas sp. (D) & ++++ & - & - & + & + & + & + & - & - & - & ++ & +++ & + & + & - & - & - & + \\
\hline P27 & Pseudomonas sp. (D) & + & + & - & + & + & + & + & + & + & - & ++ & +++ & + & + & + & - & - & + \\
\hline P30 & Pseudomonas sp. (D) & + & + & - & + & + & + & + & + & + & - & ++ & +++ & + & + & + & - & - & + \\
\hline P42 & Pseudomonas sp. (D) & + & + & $\beta$ & + & + & + & + & + & + & + & ++ & ++++ & + & + & + & - & - & + \\
\hline P116 & Pseudomonas sp. (D) & + & - & - & + & + & + & + & + & + & - & ++ & +++ & + & + & + & - & - & - \\
\hline P117 & Pseudomonas sp. (D) & + & - & - & + & + & + & + & + & - & - & ++ & +++ & + & + & + & - & - & + \\
\hline P139 & Pseudomonas sp. (D) & + & - & - & + & + & + & + & - & - & - & ++ & ++++ & + & + & + & - & - & + \\
\hline P141 & Pseudomonas sp. (D) & +++ & - & $\beta$ & + & + & + & + & + & - & - & ++ & ++++ & + & + & + & - & - & + \\
\hline P152 & Pseudomonas sp. (D) & ++ & - & - & + & + & + & + & - & - & - & ++ & ++++ & + & + & + & - & - & - \\
\hline P66 & Pseudomonas sp. (E) & + & + & - & + & + & + & + & - & - & - & ++ & +++ & + & + & + & - & - & - \\
\hline P67 & Pseudomonas sp. (E) & + & + & - & + & + & + & + & - & - & - & ++ & +++ & + & + & + & - & - & + \\
\hline P154a & Pseudomonas sp. (F) & + & - & - & + & + & + & - & - & - & - & ++ & +++ & + & + & + & - & - & + \\
\hline P154b & Pseudomonas sp. (F) & +++ & - & - & + & + & + & - & - & - & - & ++ & +++ & + & + & + & - & - & + \\
\hline P155 & Pseudomonas sp. (G) & + & - & $\beta$ & + & + & + & - & - & - & - & ++ & +++ & + & + & + & - & - & + \\
\hline $\begin{array}{l}\text { DSM } \\
12735^{\mathrm{a}}\end{array}$ & P. putida & - & - & $\alpha$ & + & + & + & + & - & - & - & ++ & +++ & - & + & + & - & - & - \\
\hline $\begin{array}{l}\text { DSM } \\
50090^{\mathrm{a}}\end{array}$ & P. fluorescens & + & - & - & + & + & + & + & - & - & - & ++ & +++ & + & + & + & - & - & - \\
\hline
\end{tabular}




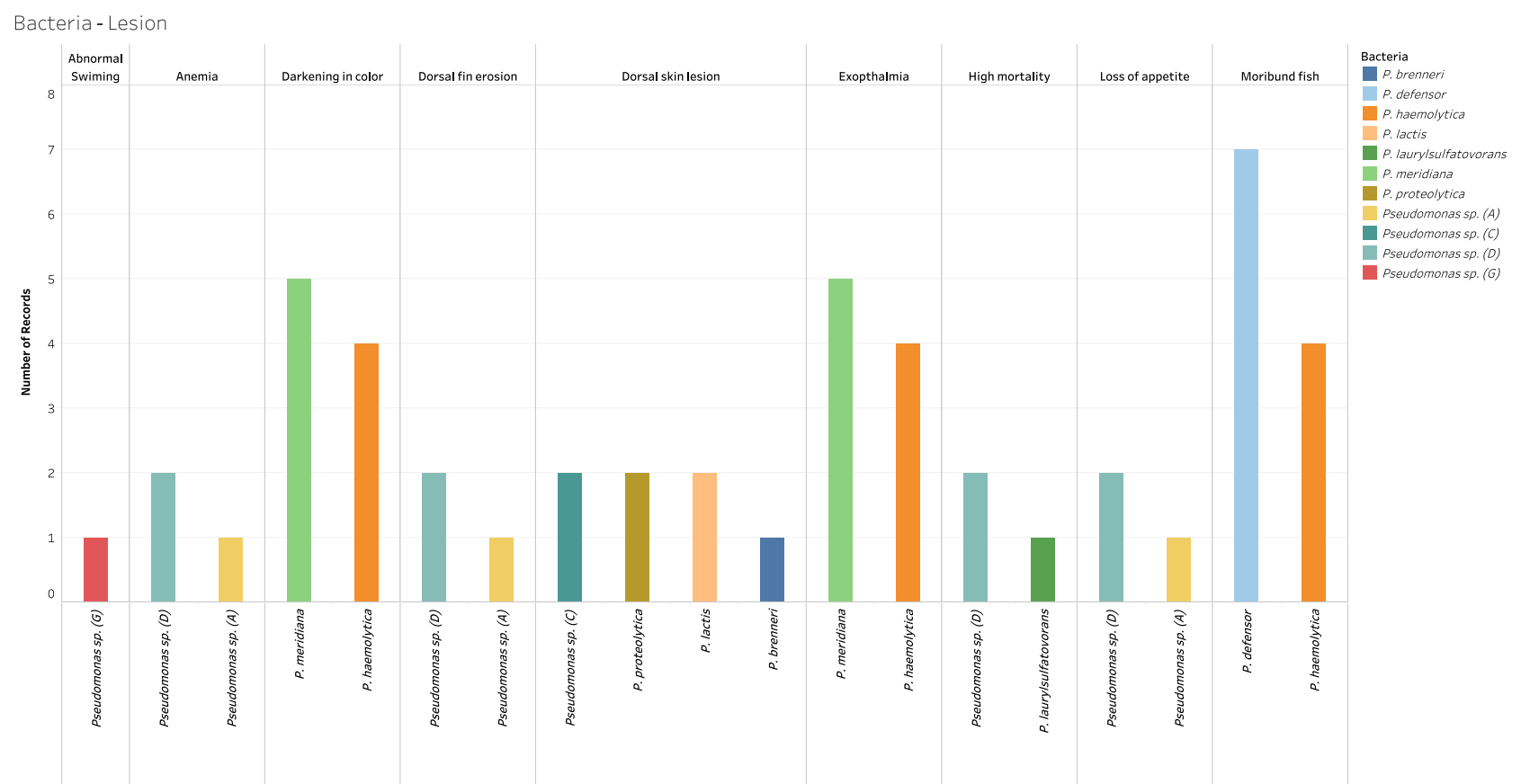

Fig. 4. Fish lesions, symptoms and Pseudomonas species correlation.

temperature (average data). $P$. lundensis had the highest tolerance to salinity, whilst $P$. migulae and $P$. laurylsulfatovorans had the lowest salt tolerance (average data) (supplementary Table 4 and supplementary Fig. 6). Also, a regression analysis was performed to determine the correlation between Pseudomonas species and fish weight. $P$. defensor, $P$. proteolytica, and $P$. haemolytica were isolated only from brood stock fish with a weight up to $3000 \mathrm{~g}$, whilst the other species were commonly isolated from small fish, from about yolk-sac up to $60 \mathrm{~g}$ (supplementary Table 3 and supplementary Fig. 1). The weights of the sampled fish are presented in supplementary Table 3 . Fish weight was indicated as " 0 " if the weight of the sampled fish was between yolk-sac to $1 \mathrm{~g}$ of fry.

As shown in Table 1 and supplementary Fig. 7; Pseudomonas spp. were accompanied by A. media and Flavobacteria spp., such that $P$. haemolytica and $P$. meridiana were commonly detected with other bacteria species. $P$. haemolytica was frequently isolated together with Aeromonas media and F. aquidurense, $F$. branchiarum, F. collinsii, F. frigidimaris, F. glaciei, F. hydatis, F. oncorhynchi, F. piscis, F. psychrophilum, F. tiangeerense, F. tructae, and Flavobacterium sp. Furthermore, P. meridiana was accompanied by the following Flavobacterium species: $F$. frigidimaris, F. piscis, F. psychrophilum, F. aquidurense and Flavobacterium sp. (Duman et al., 2018; Saticioglu et al., 2018; and unpublished data). P. brenneri, $P$. defensor, $P$. inefficax, $P$. lactis, $P$. lundensis, $P$. migulae, $P$. proteolytica, Pseudomonas sp. (A), Pseudomonas sp. (B), Pseudomonas sp. (C) and Pseudomonas sp. (E) were isolated alone with no accompanying bacteria species.

\section{Discussion}

Aquaculture farms are specific habitats, in which bacteria define economic sustainability. Bacterial water quality is a prerequisite for aquaculture, due to the strong links between the environment, fish, and humans as the ultimate consumers. The present study, aimed at performing an extensive survey of fish farms in Turkey, mainly focussed on the genus Pseudomonas, which is a very complex genus that occupies many habitats and has important functional roles in aquaculture. Pseudomonas species may show effect in many different ways: i) The presence of the pathogenic $P$. aeruginosa is an indicator of low water quality (Mena and Gerba, 2009); ii) Several Pseudomonas species have been described as fresh water fish pathogens, which are associated either with no symptom or with haemorrhage or skin and eye lesions; iii) the intensive use of antibiotics on fish farms can lead to the environmental dissemination of antibiotic-resistant Pseudomonas spp., which are of growing concern; and iv) some Pseudomonas species can be used as biocontrol or anti-pathogen agents against fungal infections (Bergan, 1981; De Bentzmann and Plésiat, 2011; Osman et al., 2012; Liu et al., 2015; Austin and Austin, 2016). To study Pseudomonas spp. in relation to these four aspects, and to establish preventive measures for the containment of potential disease outbreaks, this study was aimed at precise identification at species level.

\subsection{Species diversity}

In this study, 20 different Pseudomonas species were isolated from water, and new species were described in fish. These species are being reported for the first time in aquaculture farms in Turkey. To our knowledge, strains of the following Pseudomonas species have been isolated for the first time from freshwater salmonid fish: $P$. brenneri, $P$. lactis, $P$. lundensis, P. lurida, P. mandelii, P. migulae, $P$. meridiana, $P$. proteolytica, $P$. simiae and $P$. weihenstephanensis. One third of the isolates were identified as one of the 7 putative new Pseudomonas species, which require further taxonomic analysis: Pseudomonas sp. (A), (B), (C), (D), (E), (F) and (G).

Thirty eight percent of the identified Pseudomonas species (P. brenneri, $P$. lactis, $P$. proteolytica, $P$. haemolytica, $P$. defensor, $P$. laurylsulfatovorans, P. meridiana, Pseudomonas sp. (A), (C), (D) and (G)) were isolated from fish with disease symptoms and/or lesions. Therefore, they are considered as potential pathogens for cultured freshwater fish. The pathogenicity of these species needs to be confirmed by fulfilling Koch's postulates in fish and conducting genomic studies.

$P$. haemolytica was the species with the highest number of isolates, appearing in a higher number of aquaculture farms (Fig. 2) in different years (supplementary Fig. S5), and in both healthy and symptomatic fish of different weights (Fig. 4, supplementary Table 4). P. haemolytica strains were isolated alone or in association with other species (A. media) (Table 1). Recently, P. haemolytica has been proposed as a new species, based on the characterization of strains isolated from raw milk (Hofmann et al., 2020). The present study describes, for the first time, its presence in a different habitat, farmed fish. Overall, these data indicate 
the presence, adaptation and persistence of this bacterium in the environment (see above).

Pseudomonas sp. (B) is the only species found in farm water and also constitutes the first group of strains selected for taxonomic polyphasic study so as to be proposed as a new species (work in progress). The newly described Pseudomonas sp. (G) caused abnormal swimming (upside-down and spirally) and disease symptoms completely different from those observed with the other identified species.

This is the first step in monitoring the presence and controlling the spread of these bacteria in this particular environment.

\subsection{Geographical distribution of the species}

The most common Pseudomonas species reported from fish farms in the Black sea region of Turkey are P. putida, P. fluorescens, P. luteola, and other Pseudomonas not identified at species level. Furthermore, $P$. aeruginosa and $P$. stutzeri have been isolated in the northeast of the Mediterranean region (Matyar et al., 2010; Öztürk and Altinok, 2014; Ture et al., 2018). None of these species were isolated in the present study. Most of the Pseudomonas species were isolated from the two biggest rainbow trout hatcheries in the Aegean (Mugla) and Central Anatolia (Kayseri) regions. While P. brenneri, P. defensor, P. haemolytica, and Pseudomonas sp. (D) were isolated only from these two hatcheries (Mugla, Kayseri), P. lactis, P. laurylsulfatovorans, P. lundensis, P. migulae, and Pseudomonas sp. (A), (C), and (E) were isolated from only one fish farm (Sivas, Kayseri or Mugla).

$P$. mandelii, $P$. meridiana, and $P$. weihenstephanensis were detected in July and December in 2013, in the Aegean and Central Anatolia regions. In January 2017, P. mandelii, P. meridiana, and P. weihenstephanensis were isolated not only in the Aegean and Central Anatolia regions, but also in the Black sea region. This was attributed to the fish farms in the Black Sea region receiving stocks mostly from the Aegean and Central Anatolia regions. A similar situation was observed for bacteria isolated from fish farms located in Eastern Anatolia, such that $P$. meridiana and Pseudomonas sp. (B) were first isolated in the Aegean and Central Anatolia regions, indicating their spread to the Eastern Anatolia and Black sea regions.

\subsection{Accompanying species}

Throughout the surveillance study, in addition to the 90 Pseudomonas strains from the different regions of Turkey, we also isolated 98 motile Aeromonas (Duman et al., 2018), 137 Yersinia ruckeri (Duman et al., 2017), 25 F. psychrophilum (Saticioglu et al., 2018), 84 Flavobacterium spp. (unpublished data), and 137 Lactococcus garvieae (Duman et al., 2020) strains. The share of Pseudomonas strains among the isolated species was $15.7 \%$, and Pseudomonas was the least isolated genus of all the aquatic pathogens investigated between 2013 and 2017 (data not shown). The isolation rate of Pseudomonas compared to other species suggests an opportunistic or primarily invasive character for this genus in fish because $P$. haemolytica, $P$. laurylsulfatovorans, $P$. lurida, $P$. mandelii, P. meridiana, P. simiae, $P$. weihenstephanensis, and Pseudomonas sp. (D), (F) and (G) were isolated in association with Flavobacterium species and A. media. Pathogenicity has not been reported for Flavobacterium species (except for F. psychrophilum) (Austin and Austin, 2016). Many Pseudomonas species have been reported as opportunistic or primarily invasive pathogens (Austin and Austin, 2016). Accordingly, Pseudomonas spp. could be primary pathogens accompanied by opportunistic pathogens or vice versa. None Pseudomonas species found in this study has been described previously as fish pathogen, but 26 strains identified under 9 species have been isolated from symptomatic fishes without any accompanying microbiota (Table 1).

\subsection{Determinants of bacterial fitness}

Several phenotypic traits of the isolates were studied to demonstrate their ability to thrive in the study environment. Iron uptake systems, biofilm formation and haemolysis were three relevant pathogenicity traits detected in most of the species identified. Fluorescent pigments, which act as siderophores, were a selective criterion for isolation. Half of the isolates were able to form pellicles that could confer survival advantages, such as nutrient availability through metabolic cooperation, acquisition of new genetic traits, and protection from the environment (Kolter and Greenberg, 2006; Karatan and Watnick, 2009). None of the pellicle-forming species detected in the present study have been previously reported to have this ability. In the present study, haemolysis was detected as a virulence characteristic in 7 species. $P$. haemolytica was isolated, for the first time, from rainbow trout and all strains were strongly $\beta$-haemolytic. This species has been reported to have been isolated from raw milk and it is difficult to explain its presence in fish farms. It is considered that this could be related to the water sources being used for the provision of water to cattle, sheep, and goats, and in some regions, cattle having access to the water sources before the water reached the fish farm. These propositions could explain why $P$. haemolytica was detected in both dairy products and farmed fish as well as the detection of $P$. lactis, another species originally isolated from bovine raw milk (Von Neubeck et al., 2017). However, in addition to $P$. haemolytica and $P$. lactis, other species, including $P$. lurida, $P$. meridiana, P. weihenstephanensis, and Pseudomonas sp. (B), (C), (D) and (G) were also haemolytic, which could explain the haemorrhagic symptoms displayed by the fish they had colonized.

Pseudomonas species are known to display varying levels of tolerance to salinity, temperature and $\mathrm{pH}$, and to persist in the environment, even in the absence of nutrients (Kiran et al., 2004; Kumar et al., 2008; LaBauve and Wargo, 2012; Liu et al., 2015; Palleroni, 2015; Moradali et al., 2017). In the present study, all isolates tolerated $4.5 \% \mathrm{NaCl}$, and some were highly tolerant, up to $9 \% \mathrm{NaCl}$ (some strains of $P$. haemolytica, $P$. lundensis, $P$. proteolytica, $P$. weihenstephanensis and Pseudomonas sp. (D)). While almost all isolates could grow at an incubation temperature of $37{ }^{\circ} \mathrm{C}, P$. mandelii (P31), P. meridiana (V35 and V36), P. migulae and Pseudomonas sp. (B) tolerated $42{ }^{\circ} \mathrm{C}$, and $P$. haemolytica (V81) was heat-tolerant up to $45^{\circ} \mathrm{C}$. Furthermore, it was determined that all of the isolates were able to survive in sterile distilled water and tap water without any additives. Several of these phenotypic findings point out to the possibility of these strains being major zoonotic agents and potential pathogens for humans and terrestrial animals.

Pseudomonas spp. have been recognized as primarily invasive or opportunistic pathogens for many organisms and, furthermore, this genus has also gained importance in terms of antimicrobial resistance. Even in cases of mass death, when veterinarians prefer to use broadspectrum antimicrobials regardless of the etiological agent, it is important to review the antibiogram of the isolated strains. Several researchers have evaluated the antimicrobial sensitivity of Pseudomonas species isolated from fish, and have reported them as multidrug-resistant bacteria, based on their resistance to ampicillin, trimethoprim+sulfamethoxazole, cefotaxime, aztreonam, nitrofurantoin and other groups of antimicrobials (Matyar et al., 2008; Devarajan et al., 2017). A limited number of antimicrobial agents are licensed for the treatment of fish diseases and those that have found common use in Turkey are trimethoprim/sulfamethoxazole, doxycycline, tetracycline, enrofloxacin, and florfenicol. In the present study, we tested all Pseudomonas strains against the antimicrobials commonly used in fish farms in Turkey, and determined that almost all species were resistant to sulfonamides (SXT), aminopenicillins (AML), penicillins (AMP), macrolides (E), clindamycin (MY) and chloramphenicol (FFC). In contrast to previously published results, we found that the isolates were frequently susceptible to tetracyclines (DO, TE, and OT), quinolones (ENR, CIP, $\mathrm{UB}$ ), and an aminoglycoside (CN). As the antimicrobial resistance of the large majority of these species have not been described before, a literature-based comparison of the isolates was able to be made only to a very limited level. The comparison of the isolates showed that P. defensor, P. meridiana, and Pseudomonas sp. (E) were the most resistant 
species.

An important result of this study is the isolation of a high number of Pseudomonas species from an environment previously not described for these species. $P$. aeruginosa, an important health indicator, and the fish pathogens $P$. anguilliseptica, $P$. chlororaphis, $P$. putida and $P$. luteola have been detected neither in fish nor in water, indicating a good microbial quality of water. Only one new Pseudomonas species, namely, Pseudomonas sp. (B), was found exclusively in water and not in fish. None of the Pseudomonas species isolated in this study were considered as fish pathogens until now. Pseudomonas sp. (D) is phylogenetically close to $P$. baetica, which is considered a fish pathogen and, consequently should be tested for pathogenicity. Either the spread of the strains from one region to another has been confirmed ( $P$. mandelii, $P$. meridiana, $P$. weihenstephanensis), or the appearance of some bacteria, such as $P$. haemolytica and $P$. lactis, has been described for the first time in fish farms, which represent a totally different environment from the original source of isolation. The newly identified species were highly resistant to the antibiotics commonly used in aquaculture, and this fact should be carefully addressed in the context of the environmental dissemination of antibiotic resistance. The phenotypic and genotypic characteristics of these isolates could contribute to a better understanding of their future role, and their fitness in spreading to other regions.

\section{Conclusion}

The multilocus analyses presented is a precise method for the identification at the species level of Pseudomonas associated with water and fish in aquaculture farms. It demonstrates the high diversity of species present in this particular habitat:12 Pseudomonas species are reported for the first time associated with freshwater salmonid fish and seven not yet described putative new Pseudomonas species have been also found. The newly identified species were highly resistant to the antibiotics used in aquaculture. These data highlight the importance of continuous monitoring of the presence and spreading of microorganisms in aquaculture farms.

Supplementary data to this article can be found online at https://doi. org/10.1016/j.aquaculture.2021.736369.

\section{Funding}

This research was supported by the Scientific and Technological Research Council of Turkey (TUBITAK) [No: 1180420], and approved by the Local Ethics Commission (report 2012-14-04).

\section{CRediT authorship contribution statement}

Muhammed Duman: Methodology, Software, Data curation, Writing - original draft, Editing. Magdalena Mulet: Methodology, Software. Soner Altun: Methodology, Software, Writing - original draft. Izzet Burcin Saticioglu: Methodology, Software, Writing - original draft. Burak Ozdemir: Software. Nihed Ajmi: Software. Jorge Lalucat: Data curation, Writing - original draft. Elena García-Valdés: Data curation, Writing - original draft, Supervision, Editing.

\section{Declaration of Competing Interest}

The authors declare no competing or financial interests.

\section{Acknowledgements}

The authors would like to thank Mr. Ozgun Yilmaz for his statistical analysis and comments. We also like to thank Google Maps for the image in Fig. 1.

\section{References}

EUCAST-European Committee on Antimicrobial Susceptibility Testing, 2020 EUCAST: Clinical Breakpoints and Dosing of Antibiotics https://www.eucast.org/clinical_bre akpoints/ (accessed 6.29.20).

Ait Tayeb, L., Ageron, E., Grimont, F., Grimont, P.A.D., 2005. Molecular phylogeny of the genus Pseudomonas based on rpoB sequences and application for the identification of isolates. Res. Microbiol. 156, 763-773. https://doi.org/10.1016/j. resmic.2005.02.009.

Altinok, I., Kayis, S., Capkin, E., 2006. Pseudomonas putida infection in rainbow trout. Aquaculture 261, 850-855. https://doi.org/10.1016/j.aquaculture.2006.09.009.

Altinok, I., Balta, F., Capkin, E., Kayis, S., 2007. Disease of rainbow trout caused by Pseudomonas luteola. Aquaculture 273, 393-397. https://doi.org/10.1016/j. aquaculture.2007.10.025.

Analytics, L., 2013. Analytics 2013 A survey on analytic usage, trends, and future initiatives.

Austin, B., Austin, D.A., 2016. Pseudomonads. In: Bacterial Fish Pathogens. Springer International Publishing, pp. 475-498. https://doi.org/10.1007/978-3-319-32674$0 \_9$.

Austin, B., Newaj-Fyzul, A. (Eds.), 2017. Diagnosis and Control of Diseases of Fish and Shellfish, Diagnosis and Control of Diseases of Fish and Shellfish. John Wiley \& Sons, Ltd, Chichester, UK. https://doi.org/10.1002/9781119152125.

Baumann, P., Bowditch, R.D., Baumann, L., Beaman, B., 1983. Taxonomy of marine Pseudomonas species: P. stanieri, sp. nov.; P. perfectomarina sp. nov., nom. Rev.; P. nautica; and P. doudoroffii. Int. J. Syst. Bacteriol. 33, 857-865. https://doi.org/ 10.1099/00207713-33-4-857.

Bergan, T., 1981. Human- and animal-pathogenic members of the genus pseudomonas. In: The Prokaryotes. Springer, Berlin Heidelberg, pp. 666-700. https://doi.org/ 10.1007/978-3-662-13187-9_59.

CLSI, 2017. Performance Standards for Antimicrobial Susceptibility Testing, Performance Standards for Antimicrobial Susceptibility Testing.

CLSI - Clinical and Laboratory Standards Institute, 2006. VET03-a Methods for Antimicrobial Disk Susceptibility Testing of Bacteria Isolated from Aquatic Animals; Approved Guideline A Guideline for Global Application Developed Through the Clinical and Laboratory Standards Institute Consensus Process.

CLSI - Clinical and Laboratory Standards Institute, 2014. Performance Standards for Antimicrobial Susceptibility Testing of Bacteria Isolated from Aquatic Animals; Second Informational Supplement. Clin. Lab. Stand, Institute, PA.

Das, B.K., Samal, S.K., Samantaray, B.R., Sethi, S., Pattnaik, P., Mishra, B.K., 2006. Antagonistic activity of cellular components of Pseudomonas species against Aeromonas hydrophila. Aquaculture 253, 17-24. https://doi.org/10.1016/j. aquaculture.2005.01.028.

De Bentzmann, S., Plésiat, P., 2011. The Pseudomonas aeruginosa opportunistic pathogen and human infections. Environ. Microbiol. 13, 1655-1665. https://doi. org/10.1111/j.1462-2920.2011.02469.x.

Derome, N., Gauthier, J., Boutin, S., Llewellyn, M., 2016. The rasputin effect: when commensals and symbionts become parasitic. Adv. Environ. Microbiol. https://doi. org/10.1007/978-3-319-28170-4.

Devarajan, N., Köhler, T., Sivalingam, P., van Delden, C., Mulaji, C.K., Mpiana, P.T., Ibelings, B.W., Poté, J., 2017. Antibiotic resistant Pseudomonas spp. in the aquatic environment: a prevalence study under tropical and temperate climate conditions. Water Res. 115, 256-265. https://doi.org/10.1016/j.watres.2017.02.058.

Duman, M., Altun, S., Cengiz, M., Saticioglu, I.B., Buyukekiz, A.G., Sahinturk, P., 2017. Genotyping and antimicrobial resistance genes of Yersinia ruckeri isolates from rainbow trout farms. Dis. Aquat. Org. 125 https://doi.org/10.3354/dao03132.

Duman, M., Saticioglu, I.B., Janda, J.M., Altun, S., 2018. The determination of the infectious status and prevalence of motile Aeromonas species isolated from disease cases in rainbow trout (Oncorhynchus mykiss) and aquarium fish. J. Fish Dis. 41, 1843-1857. https://doi.org/10.1111/jfd.12896.

Duman, M., Buyukekiz, A.G., Saticioglu, I.B., Cengiz, M., Sahinturk, P., Altun, S., 2020. Epidemiology, genotypic diversity, and antimicrobial resistance of Lactococcus garvieae in farmed rainbow trout Oncorhynchus mykiss. Iran. J. Fish. Sci. 19, 1-18. https://doi.org/10.22092/ijfs.2018.117609.

Felsenstein, J., 1981. Evolutionary trees from DNA sequences: a maximum likelihood approach. J. Mol. Evol. 17, 368-376. https://doi.org/10.1007/BF01734359.

Gomila, M., Peña, A., Mulet, M., Lalucat, J., García-Valdés, E., 2015. Phylogenomics and systematics in Pseudomonas. Front. Microbiol. 6, 1-13. https://doi.org/10.3389/ fmicb.2015.00214.

Hofmann, K., Huptas, C., Doll, E.V., Scherer, S., Wenning, M., Wenning, M., 2020. Pseudomonas haemolytica sp. nov., isolated from raw milk and skimmed milk concentrate. Int. J. Syst. Evol. Microbiol. 70, 2339-2347. https://doi.org/10.1099/ ijsem.0.004043.

Jukes, T., Cantor, C., 1969. Evolution of protein molecules. In: Munro (Ed.), New York: Academic Press. ACADEMIC PRESS, INC. https://doi.org/10.1016/B978-1-48323211-9.50009-7

Karatan, E., Watnick, P., 2009. Signals, regulatory networks, and materials that build and break bacterial biofilms. Microbiol. Mol. Biol. Rev. 73, 310-347. https://doi.org/ 10.1128/mmbr.00041-08.

Khan, N.H., Ahsan, M., Taylor, W.D., Kogure, K., 2010. Culturability and survival of marine, freshwater and clinical Pseudomonas aeruginosa. Microbes Environ. 25, 266-274. https://doi.org/10.1264/jsme2.ME09178.

Kiran, M.D., Prakash, A.J.S.S., Annapoorni, A.S., Dube, S., Kusano, A.T., Okuyama, A.H., Murata, A.N., Shivaji, S., 2004. Psychrophilic Pseudomonas Syringae Requires TransMonounsaturated Fatty Acid for Growth at Higher Temperature. Extremophiles 8 401-410. https://doi.org/10.1007/s00792-004-0401-8. 
Kolter, R., Greenberg, E.P., 2006. Microbial sciences: the superficial life of microbes. Nature 441, 300-302. https://doi.org/10.1038/441300a.

Kumar, M., León, V., De Sisto Materano, A., Ilzins, O.A., Luis, L., 2008. Biosurfactant production and hydrocarbon-degradation by halotolerant and thermotolerant Pseudomonas sp. World J. Microbiol. Biotechnol. 24, 1047-1057. https://doi.org/ 10.1007/s11274-007-9574-5.

LaBauve, A.E., Wargo, M.J., 2012. Growth and laboratory maintenance of Pseudomonas aeruginosa. Curr. Protoc. Microbiol. 1-8 https://doi.org/10.1002/9780471729259. mc06e01s 25 .

Lamichhane, J.R., Varvaro, L., 2013. A new medium for the detection of fluorescent pigment production by pseudomonads. Plant Pathol. 62, 624-632. https://doi.org/ 10.1111/j.1365-3059.2012.02670.x.

Liu, Y., Rzeszutek, E., Van Der Voort, M., Wu, C.H., Thoen, E., Skaar, I., Bulone, V., Dorrestein, P.C., Raaijmakers, J.M., De Bruijn, I., 2015. Diversity of aquatic Pseudomonas species and their activity against the fish pathogenic oomycete Saprolegnia. PLoS One 10, 1-17. https://doi.org/10.1371/journal.pone.0136241.

Loch, T.P., Fujimoto, M., Woodiga, S.A., Walker, E.D., Marsh, T.L., Faisal, M., 2013. Diversity of fish-associated flavobacteria of Michigan. J. Aquat. Anim. Health 25, 149-164. https://doi.org/10.1080/08997659.2012.758189.

López, J.R., Diéguez, A.L., Doce, A., de la Roca, E., de la Herran, R., Navas, J.I., Toranzo, A.E., Romalde, J.L., 2012. Pseudomonas baetica sp. nov., a fish pathogen isolated from wedge sole, Dicologlossa cuneata (Moreau). Int. J. Syst. Evol. Microbiol. 62, 874-882. https://doi.org/10.1099/ijs.0.030601-0.

Matyar, F., Kaya, A., Dinçer, S., 2008. Antibacterial agents and heavy metal resistance in gram-negative bacteria isolated from seawater, shrimp and sediment in Iskenderun Bay, Turkey. Sci. Total Environ. 407, 279-285. https://doi.org/10.1016/j. scitotenv.2008.08.014.

Matyar, F., Akkan, T., Uçak, Y., Eraslan, B., 2010. Aeromonas and Pseudomonas: antibiotic and heavy metal resistance species from Iskenderun Bay, Turkey (Northeast Mediterranean Sea). Environ. Monit. Assess. 167, 309-320. https://doi. org/10.1007/s10661-009-1051-1.

Mena, K.D., Gerba, C.P., 2009. Risk assessment of pseudomonas aeruginosa in water. Rev. Environ. Contam. Toxicol. 201, 71-115. https://doi.org/10.1007/978-1-44190032-6_3.

Moradali, M.F., Ghods, S., Rehm, B.H.A., 2017. Pseudomonas aeruginosa lifestyle: a paradigm for adaptation, survival, and persistence. Front. Cell. Infect. Microbiol. 7 https://doi.org/10.3389/fcimb.2017.00039.

Mulet, M., Bennasar, A., Lalucat, J., García-Valdés, E., 2009. An rpoD-based PCR procedure for the identification of Pseudomonas species and for their detection in environmental samples. Mol. Cell. Probes 23, 140-147. https://doi.org/10.1016/j. mcp.2009.02.001.

Mulet, M., Gomila, M., Scotta, C., Sánchez, D., Lalucat, J., García-Valdés, E., 2012. Concordance between whole-cell matrix-assisted laser-desorption/ionization timeof-flight mass spectrometry and multilocus sequence analysis approaches in species discrimination within the genus Pseudomonas. Syst. Appl. Microbiol. 35, 455-464. https://doi.org/10.1016/j.syapm.2012.08.007.

Mulet, M., Gomila, M., Ramírez, A., Cardew, S., Moore, E.R.B., Lalucat, J., GarcíaValdés, E., 2017. Uncommonly isolated clinical Pseudomonas: identification and phylogenetic assignation. Eur. J. Clin. Microbiol. Infect. Dis. 36, 351-359. https:// doi.org/10.1007/s10096-016-2808-4.
Nei, M., Kumar, S., 2000. Molecular Evolution and Phylogenetics. Oxford University Press, New York.

Nixon, G.M., Armstrong, D.S., Carzino, R., Carlin, J.B., Olinsky, A., Robertson, C.F., Grimwood, K., 2001. Clinical outcome after early Pseudomonas aeruginosa infection in cystic fibrosis. J. Pediatr. 138, 699-704. https://doi.org/10.1067/ mpd.2001.112897.

Oh, W.T., Jun, J.W., Giri, S.S., Yun, S., Kim, H.J., Kim, S.G., Kim, S.W., Kang, J.W., Han, S.J., Kwon, J., Kim, J.H., Smits, T.H.M., Park, S.C., 2019. Pseudomonas tructae sp. Nov., novel species isolated from rainbow trout kidney. Int. J. Syst. Evol. Microbiol. 69, 3851-3856. https://doi.org/10.1099/ijsem.0.003696.

OIE-Office International Des E, 2000. Diagnostic manual for aquatic animal diseases. In: Diagnostic Manual for aqUatic Animal Diseases, Third. ed. Office International Des Epizooties, France, Paris.

Osman, K.M., Ata, N.S., Hedia, R.H., Elnaga Abu, A.S.M., El-Hariri, M., Aly, M.A.K., 2012. Emergence of an antimicrobial resistant Pseudomonas aeruginosa from human and animal clinical samples: a zoonotic and public health hazard. Glob. Vet. 9, 745-751. https://doi.org/10.5829/idosi.gv.2012.9.6.1102.

Öztürk, R.Ç., Altinok, I., 2014. Türkiye'de Göülen Bakteriyel ve Viral Balık Hastalıkları. Turkish J. Fish. Aquat. Sci. https://doi.org/10.4194/1303-2712-v14_130.

Palleroni, N.J., 2015. Pseudomonas, Bergey's Manual of Systematics of Archaea and Bacteria. John Wiley \& Sons, Inc., North Caldwell NJ, USA https://doi.org/10.1002/ 9781118960608.gbm01210.

Pridgeon, J., 2012. Major bacterial diseases in aquaculture and their vaccine development. CAB Rev. Perspect. Agric. Vet. Sci. Nutr. Nat. Resour. 7 https://doi. org/10.1079/PAVSNNR20127048.

Saticioglu, I.B., Duman, M., Wiklund, T., Altun, S., 2018. Serological and genetic characterization of Flavobacterium psychrophilum isolated from farmed salmonids in Turkey. J. Fish Dis. 41, 1899-1908. https://doi.org/10.1111/jfd.12901.

Thomas, J., Thanigaivel, S., Vijayakumar, S., Acharya, K., Shinge, D., Seelan, T.S.J., Mukherjee, A., Chandrasekaran, N., 2014. Pathogenecity of Pseudomonas aeruginosa in Oreochromis mossambicus and treatment using lime oil nanoemulsion. Colloids Surf. B Biointerfaces 116, 372-377. https://doi.org/10.1016/j. colsurfb.2014.01.019.

Ture, M., Misir, D.S., Altuntas, C., Kutlu, I., 2018. A survey of some bacterial fish pathogens on whiting (Merlangius merlanguseuxinus) in eastern Black Sea coast, Turkey. Turkish J. Fish. Aquat. Sci. 18, 1325-1329. https://doi.org/10.4194/13032712-v18_11_09.

Vazquez, S.C., Rios Merino, L., MacCormack, W.P., Fraile, E.R., 1995. Proteaseproducing psychrotrophic bacteria isolated from Antarctica. Polar Biol. 15, 131-135. https://doi.org/10.1007/BF00241051.

Von Neubeck, M., Huptas, C., Glück, C., Krewinkel, M., Stoeckel, M., Stressler, T., Fischer, L., Hinrichs, J., Scherer, S., Wenning, M., 2017. Pseudomonas lactis sp. nov. and Pseudomonas paralactis sp. nov., isolated from bovine raw milk. Int. J. Syst. Evol. Microbiol. 67, 1656-1664. https://doi.org/10.1099/ijsem.0.001836.

Yoon, Y.K., Kim, H.A., Ryu, S.Y., Lee, E.J., Lee, M.S., Kim, J., Park, S.Y., Yang, K.S., Kim, S.W., 2017. Tree-structured survival analysis of patients with Pseudomonas aeruginosa bacteremia: a multicenter observational cohort study. Diagn. Microbiol. Infect. Dis. 87, 180-187. https://doi.org/10.1016/j.diagmicrobio.2016.10.008. 